\title{
FERMILAB-SLIDES-21-102-AD-APC
}

This manuscript has been authored by Fermi Research Alliance LLC under Contract No. DE-AC02-07CH11359 with the U.S.

Department of Energy, Office of Science, Office of High Energy

-

槒
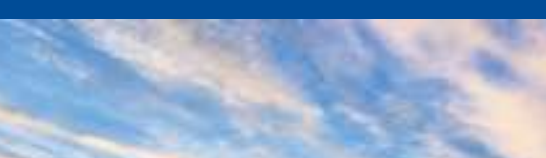

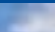
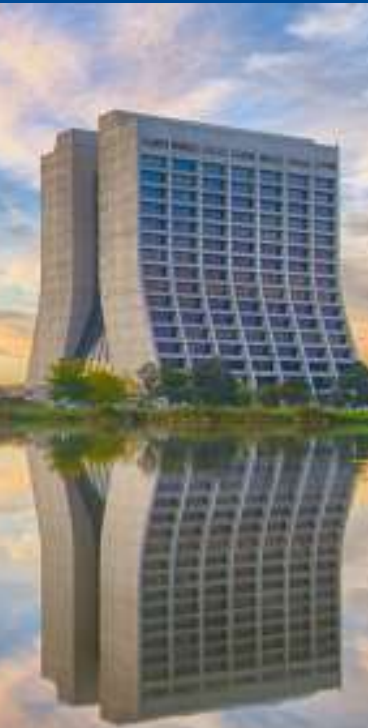

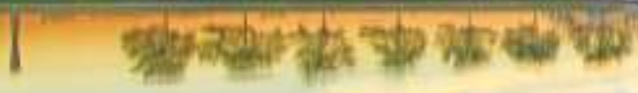

\section{Accelerator Technologies and Science: Progress and Outlook}

Vladimir SHILTSEV (Fermilab)

PANIC'2021

September 10, 2021

\section{PANIC Lisbon Portugal}

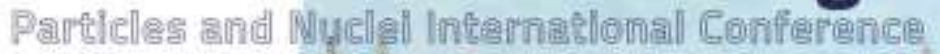

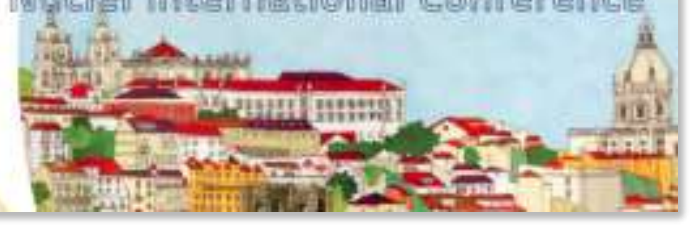




\section{Accelerators for NP}

Outstanding success of STAR/PHERNIX and ALICE operations at RHIC and LHC - since 2017:

RHIC: $>10 \mathrm{nb}-1$ 4-100 GeV/u ions ( $\mathrm{Au}, \mathrm{Zr}, \ldots$ )

RHIC: $>0.5 \mathrm{fb}-1$ in $510 \mathrm{GeV}$ cme polarized $p p$ ( $P=55 \%)$

ALICE: 1nb-1 in 5TeV cme Pb-Pb, 0.3ub-1 in Xe-Xe (comparable luminosity also to CMS, ATLAS and LHCb)

Continuous Electron Beam Accelerator Facility (CEBAF) at TJNAF : $12 \mathrm{GeV}$ electron beam energy upgrade (cw SRF linac)

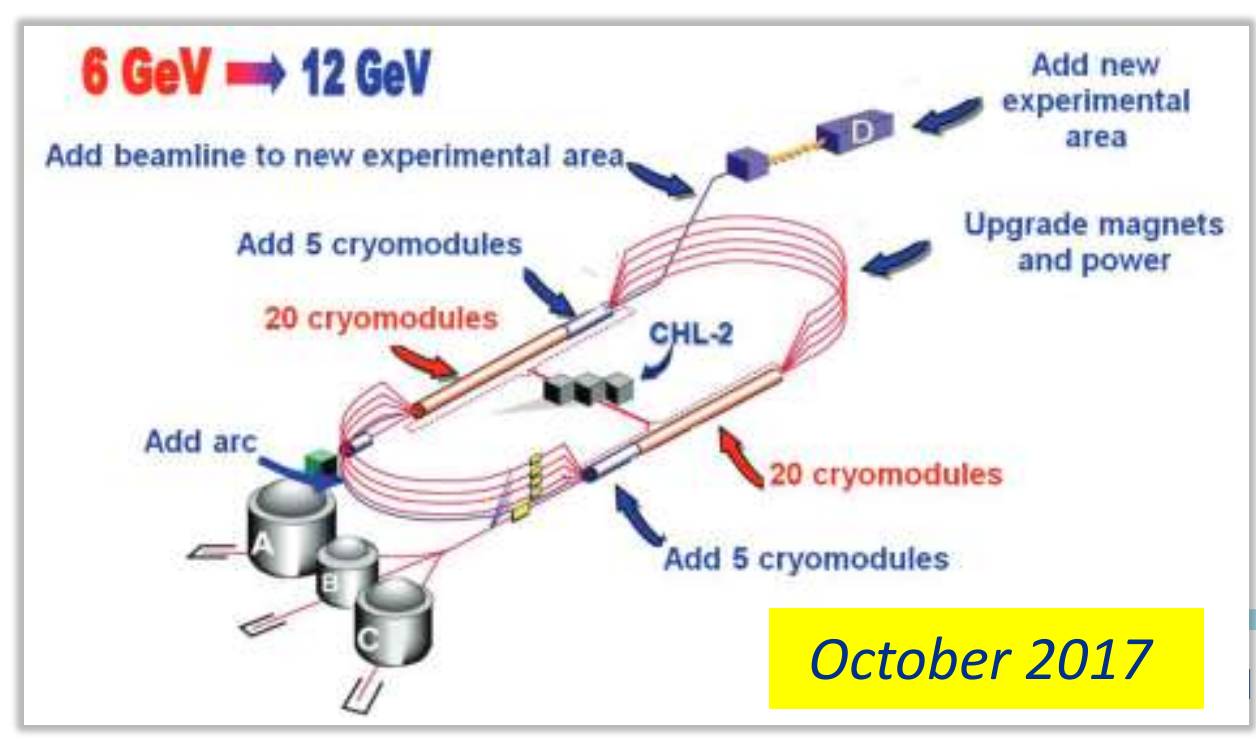

Advanced Rare Isotope Laboratory (ARIEL) at TRIUMF : $30 \mathrm{MeV} 10$ $\mathrm{mA}$ cw SRF electron linac

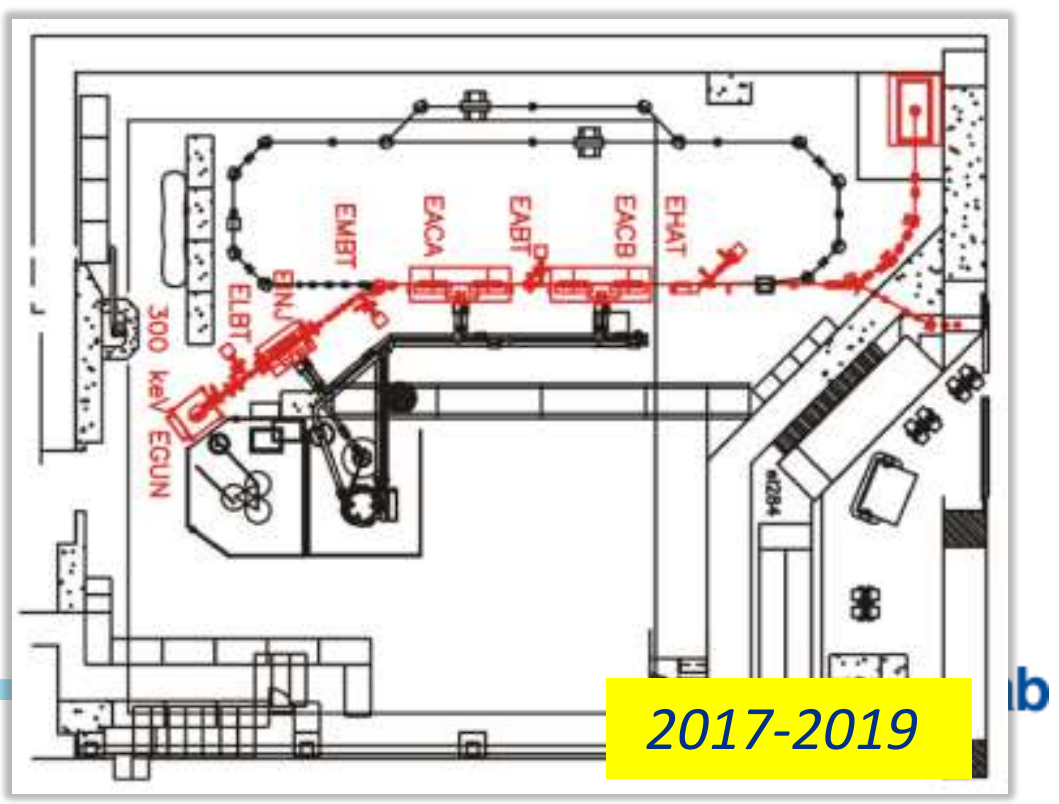




\section{Facility for Rare Isotope Beams (FRIB)}

- $200 \mathrm{MeV} / \mathrm{u}$, variety of ions Superconducting RF (80-322 MHz)

- Key Feature is $400 \mathrm{~kW}$ beam power $\left(5 \times 10^{13} 238 \mathrm{U} / \mathrm{s}\right)$ Apr. 2021: all 46 CMs $212 \mathrm{MeV} / \mathrm{u}$

Separation of isotopes "In-flight"

Suited for all elements and short half-lives

Fast, stopped, and

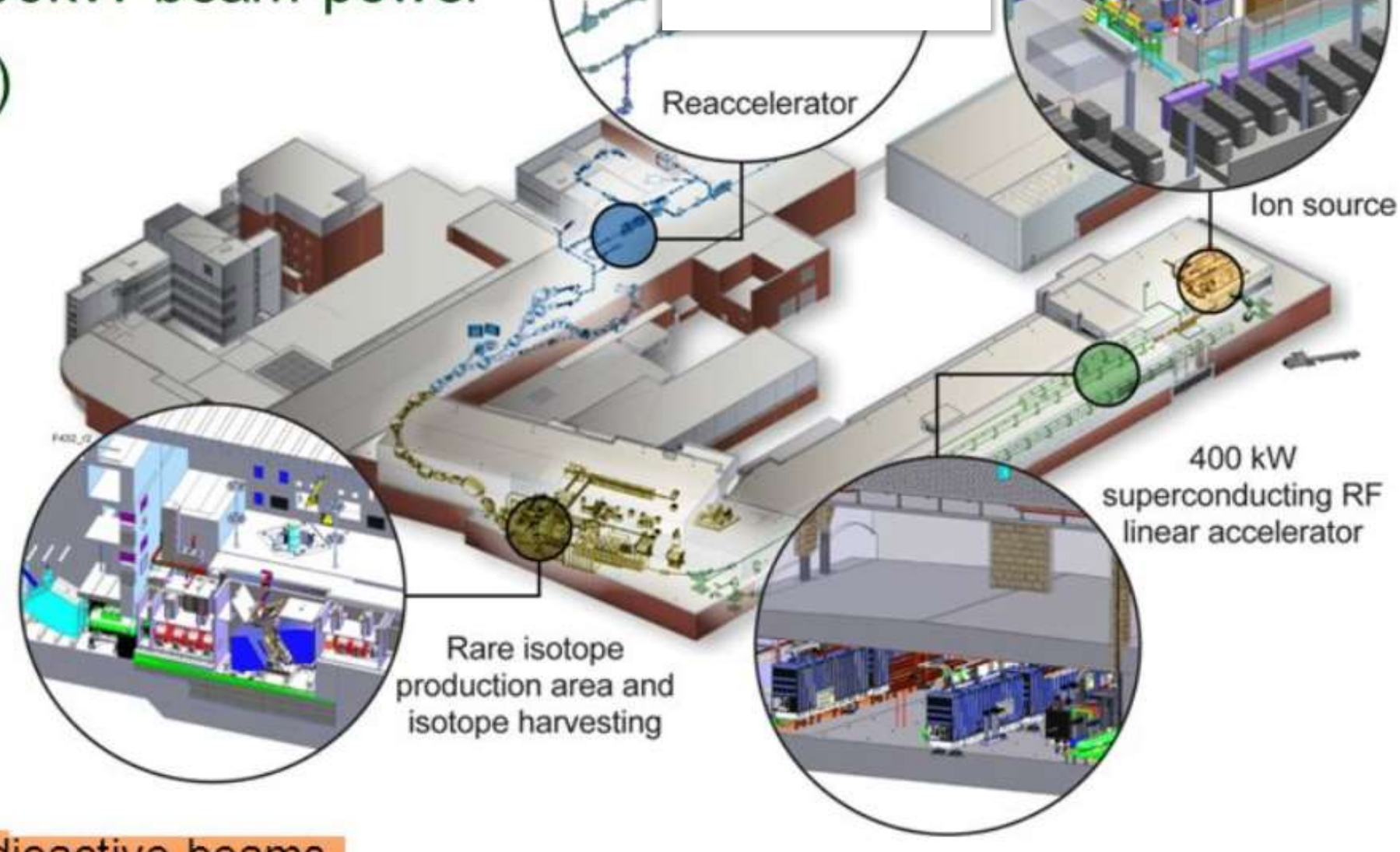
reaccelerated radioactive beams 
Facility for Antiproton and Ion Research (FAIR@GSI)

\section{FAIR}

FAIR - Facility for Antiproton and Ion Research in Europe

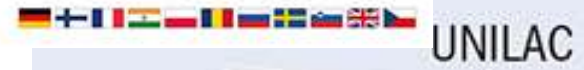

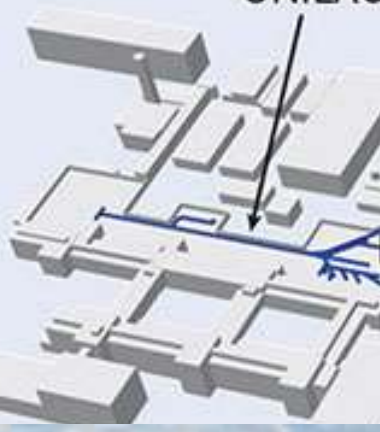

$\mathrm{p}$-Linac

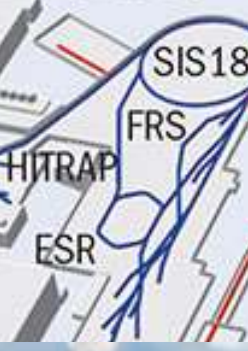

HESR

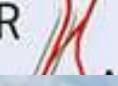

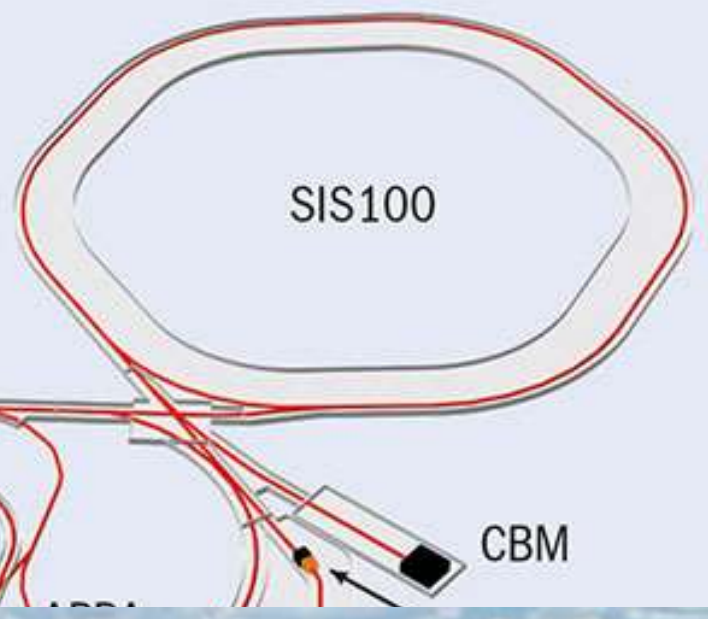

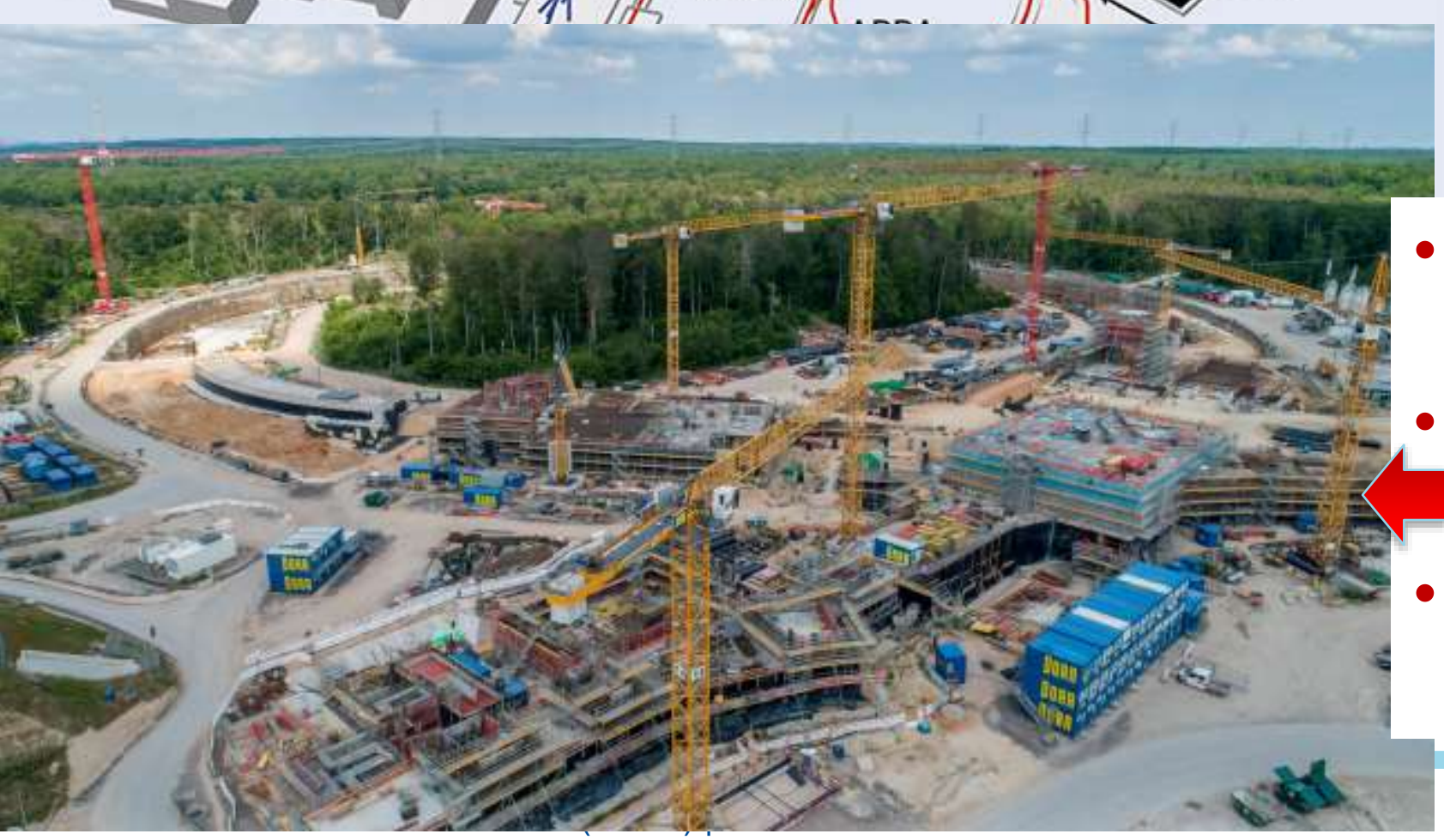

- Complex of rings and beamlines

- SIS-100: $C=1.1$ $\mathrm{km}, 29 \mathrm{GeV} p$, $2.9 \mathrm{GeV} / \mathrm{u}$ ions, SC superferric 1.9 T magnets

Groundbreaking in 2017

- Impressive civil construction SIS-100 machine installation in 2022 FFermilab 
Electron Ion Collider (EIC)

\section{Brookhaven National Laboratory}

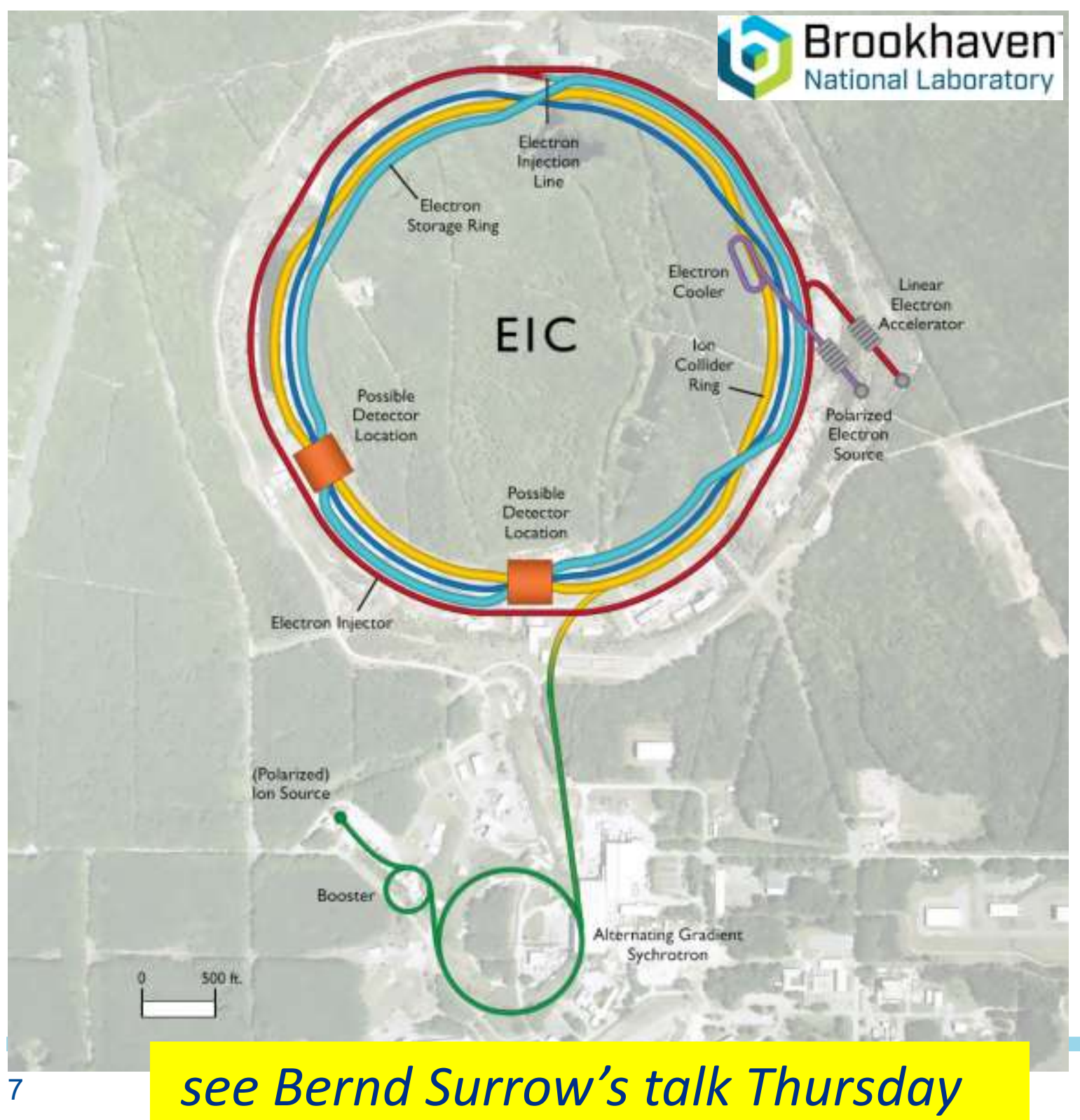

- $275 \mathrm{GeV}$ protons, $100 \mathrm{GeV} / \mathrm{u}$ (existing RHIC, upgraded)

- $10 \mathrm{GeV}$ electrons (5$18 \mathrm{GeV}$ storage ring, new)

$\sqrt{s}=20 \mathrm{GeV}$ to $100 \mathrm{GeV}$

- $~ 70 \%$ polarization

- Luminosity $\sim 100$ HERA (with Strong Hadron Cooling)

- CD-1 in July 2021

- Construction is expected to begin in 2024

- Operations early in the next decade. 


\section{Revolution in Light Sources /X-ray Sources}

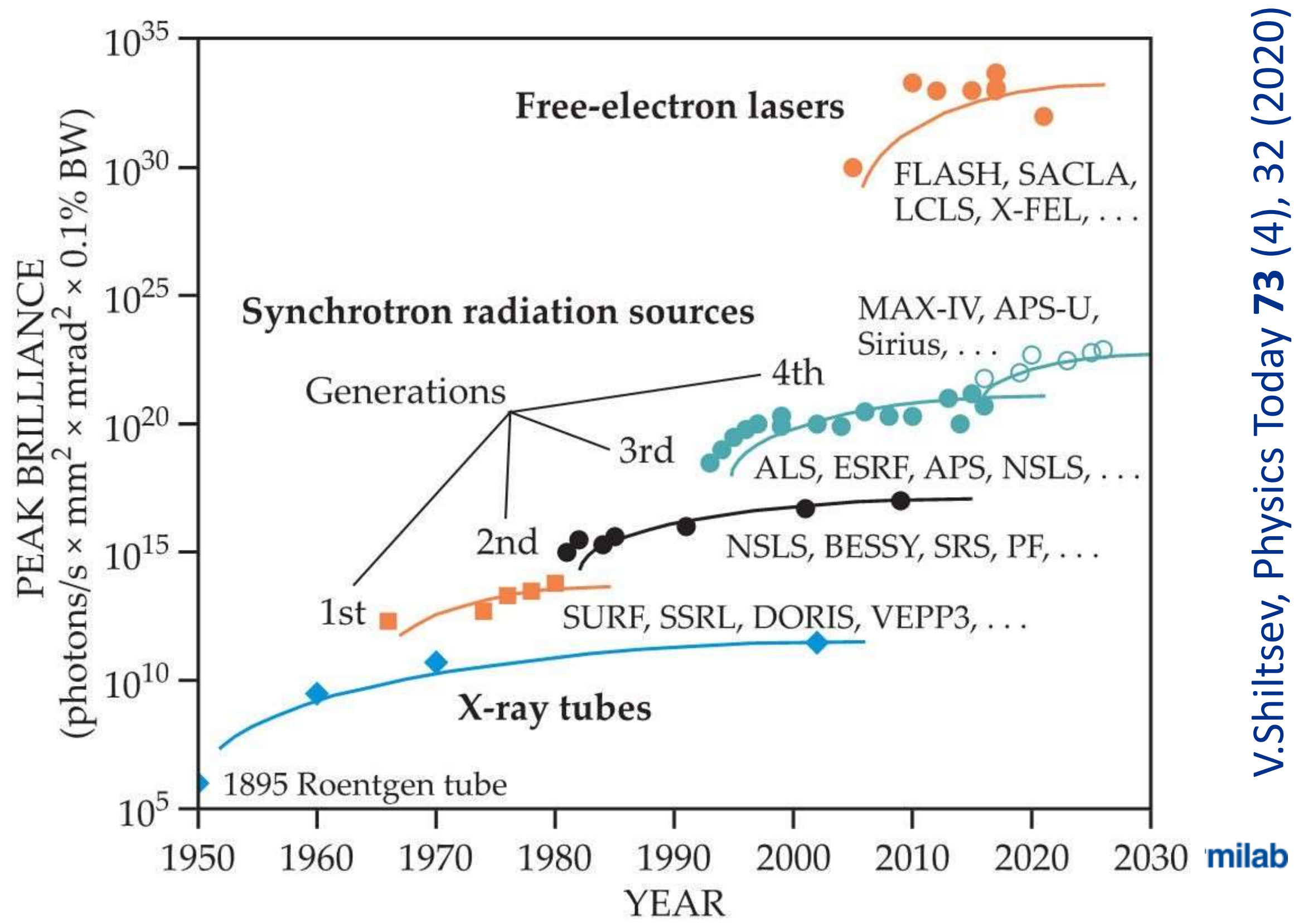




\section{$4^{\text {th }}$ Generation Light Sources aka diffraction-limited storage rings}
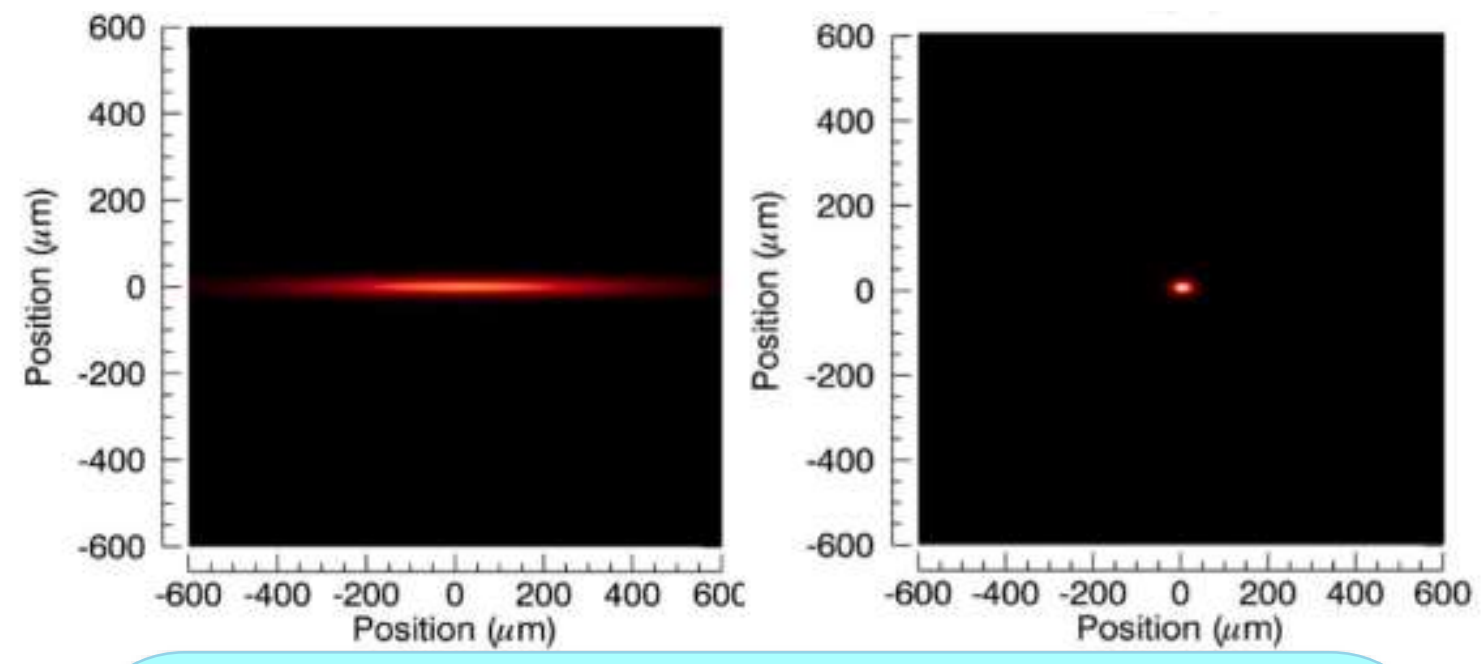

2024 -APS-Upgrade @ Argonne 6 GeV, 70 pm

2024 -SKIF @ Novosibirsk 3 GeV, 75 pm

2025 - SLS @ Swiss-PSI 2.7 GeV, 135 pm

2026 - ALS-Upgrade @ Berkeley, 2 GeV, 70 pm

2026 - HEPS @ Beijing 6 GeV, 60 pm

2027-HALF@ Hefei 2.2.GeV, 85 pm

9 2027 -PETRA-IV @ Hamburg 6 GeV, 8 pm
"Multi-Band Achromat" (MBA) advanced beam optics lattice $\rightarrow$ $x 100$ brightness increase $(1996) \rightarrow$

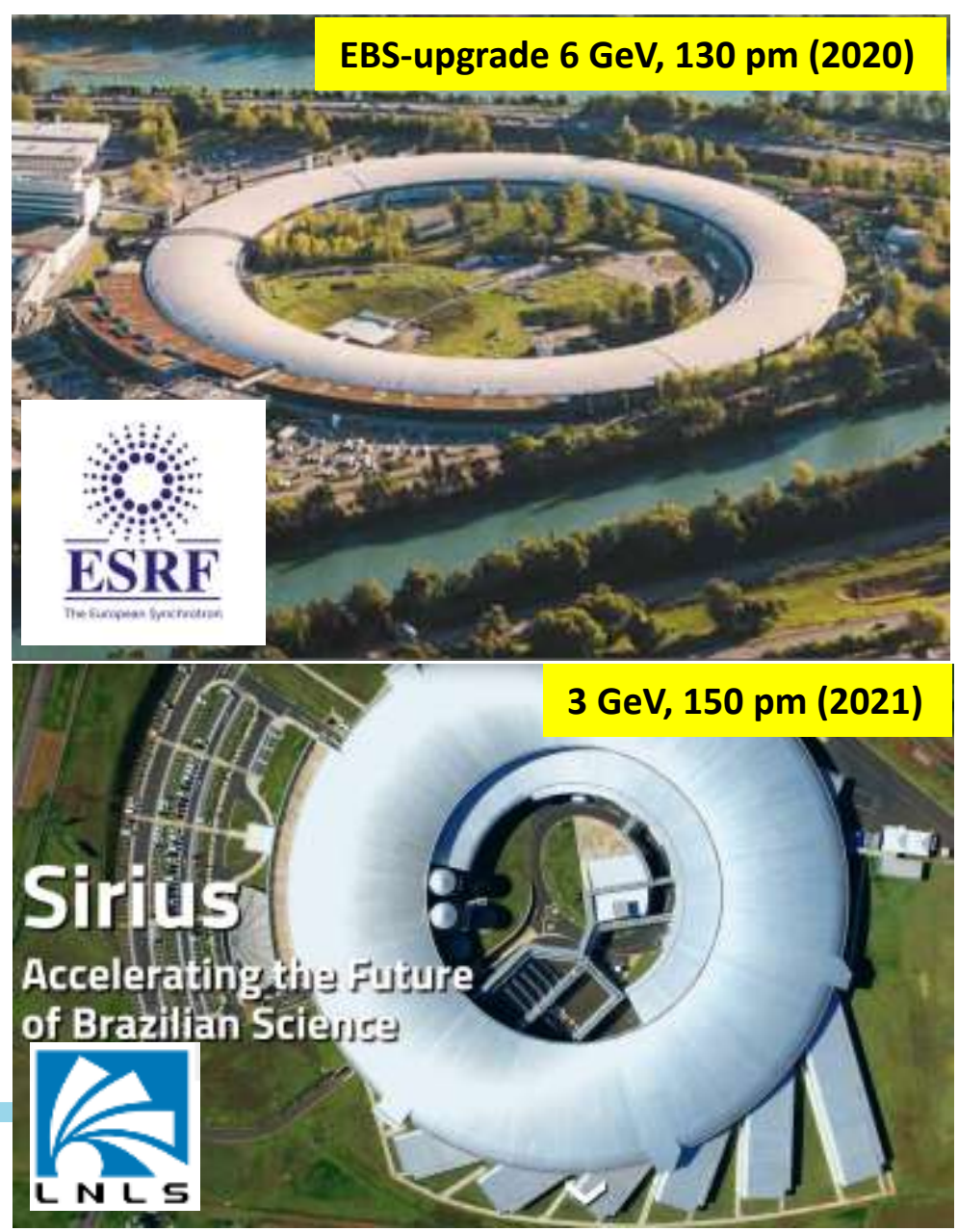


Self-Amplified Spontaneous Emission (SASE)

\section{Free Electron Lasers (FEL) \\ aka}

\section{X-FELs}

SASE्E口味L

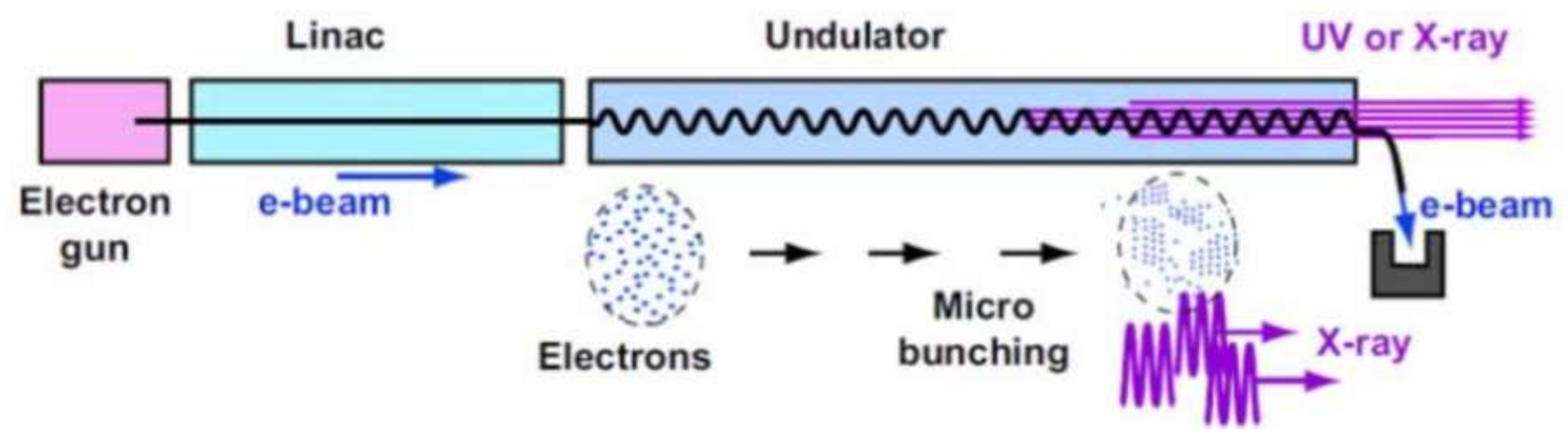

- High energy (0.1-10's of GeV) AND High brightness electron beam

- Exponential growth of radiation power while in (10's of $\mathrm{m}$ ) undulator

- Proposed in 1980, proof-of-principle demonstrations 1985-1998 


\section{XFELS}
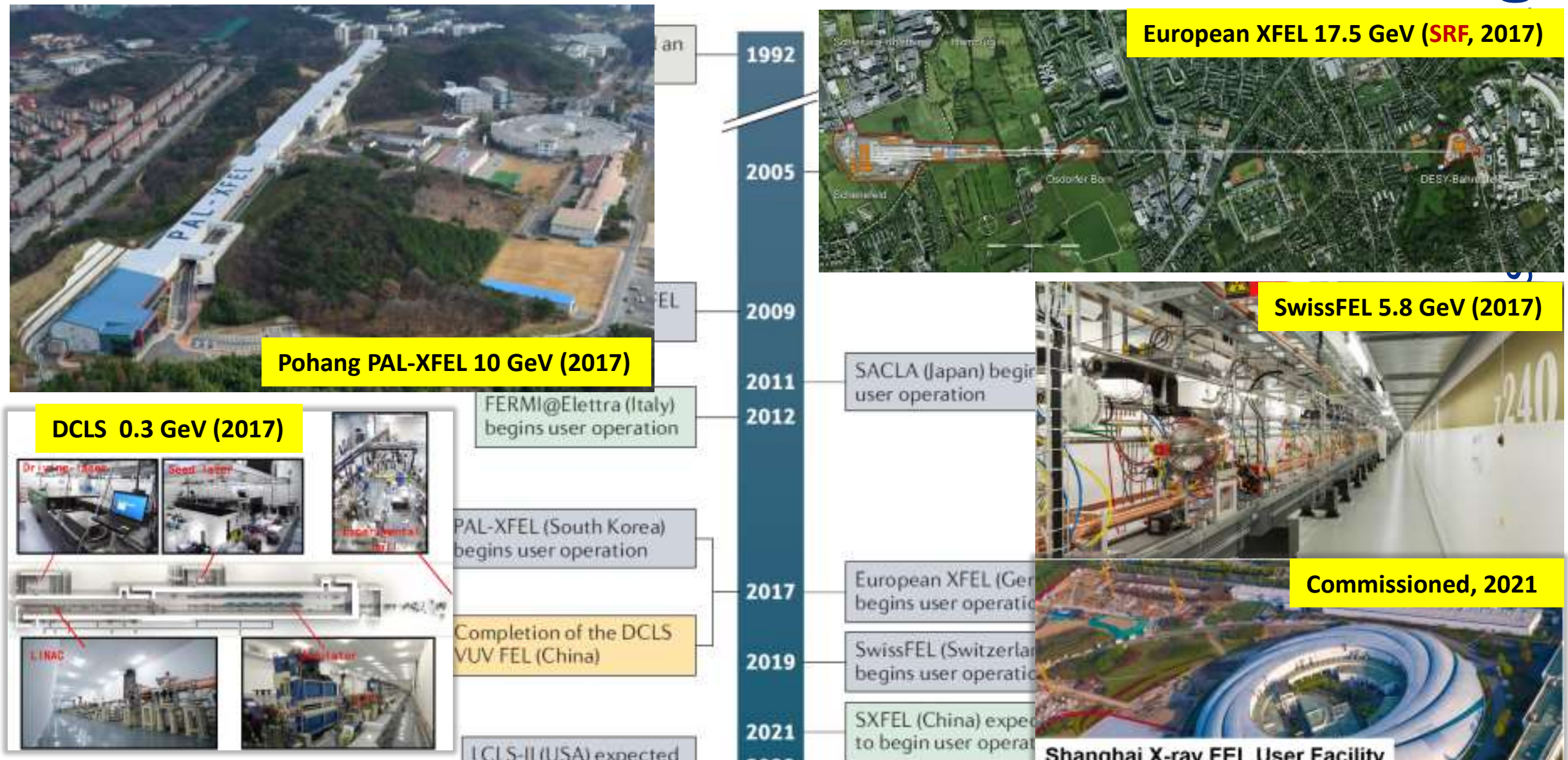

2012

PAL-XFEL (South Korea) begins user operation

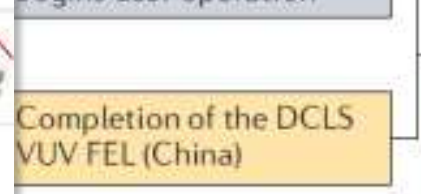

Completion of the
VUV FEL (China)

LCLS-II (USA) expected

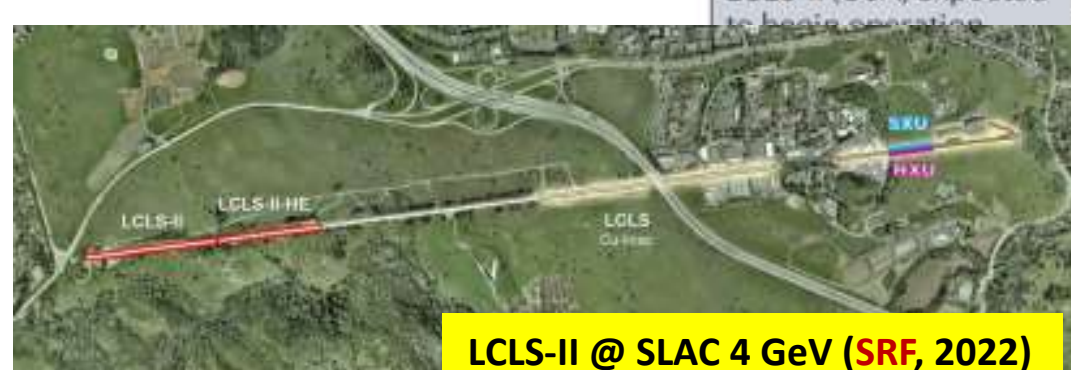

2017

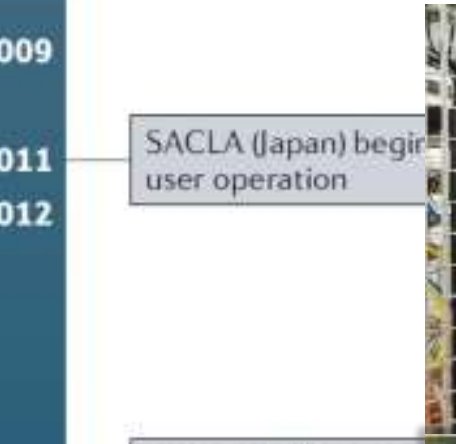

(i) $15 \times$

SwissFEL 5.8 GeV (2017)

it.

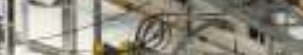

European XFEL IGer

begins user operatic
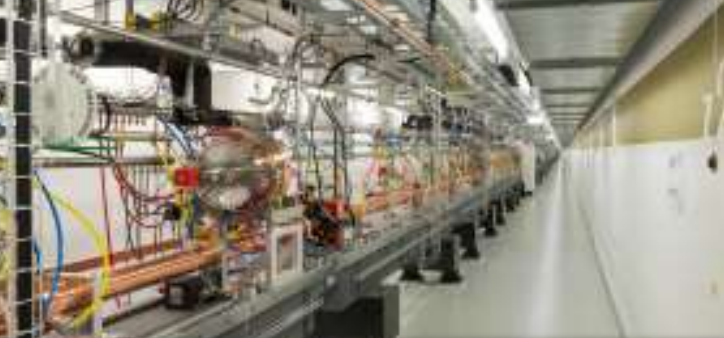

(3)
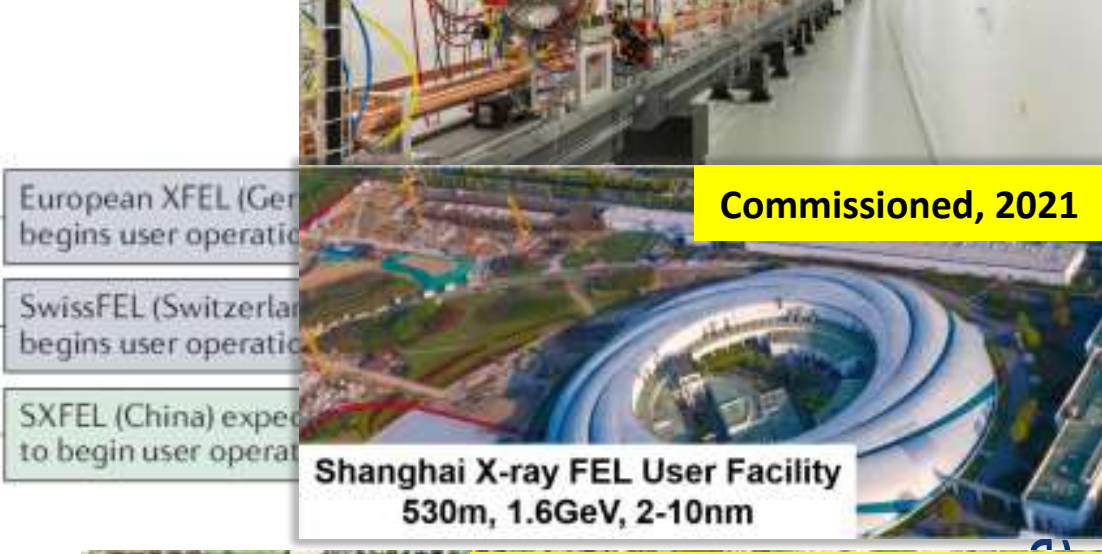

2021

2022

e-beamr $8 \mathrm{GeV}$

Commissioned, 2021

SHIN Photon enengy: $0.4-25 \mathrm{keV}$.

Shanghai SHINE 8 GeV (SRF, 2025)

to Pulse duration : 1-100t

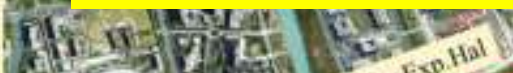

Total length: $3.1 \mathrm{~km}$

ca $30 \mathrm{~m}$ underground .

yradres

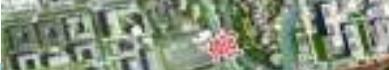




\section{Neutrino Superbeams - v Oscillations}

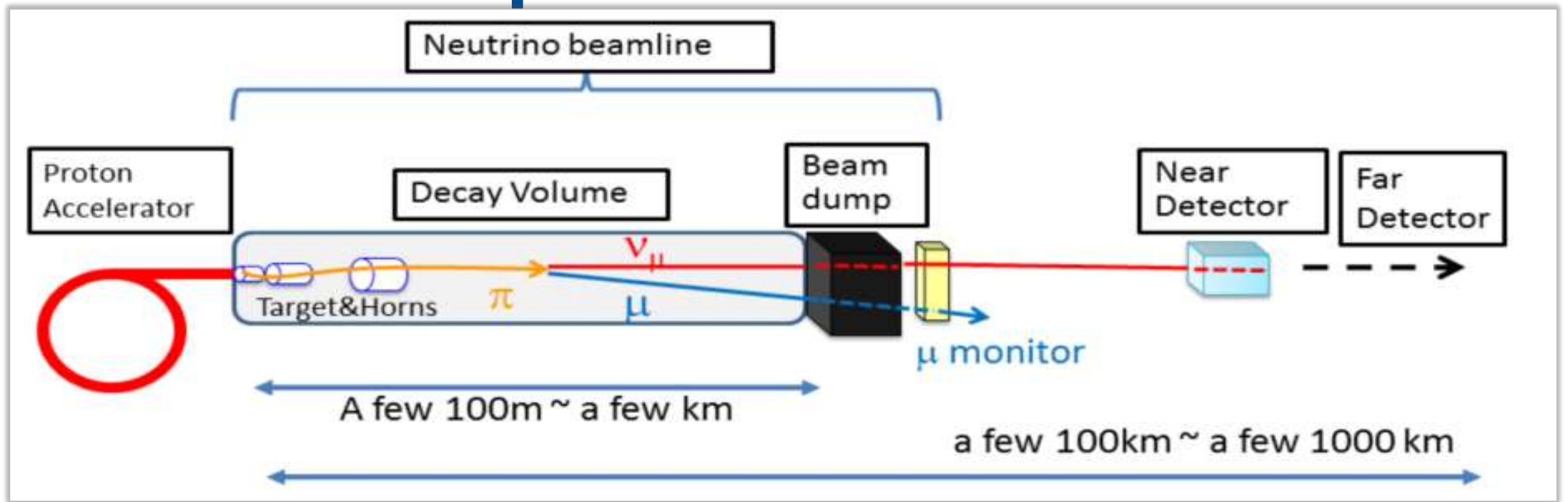

Japan Proton Accelerator Research Complex - 3/30 GeV (295 km to SuperK)

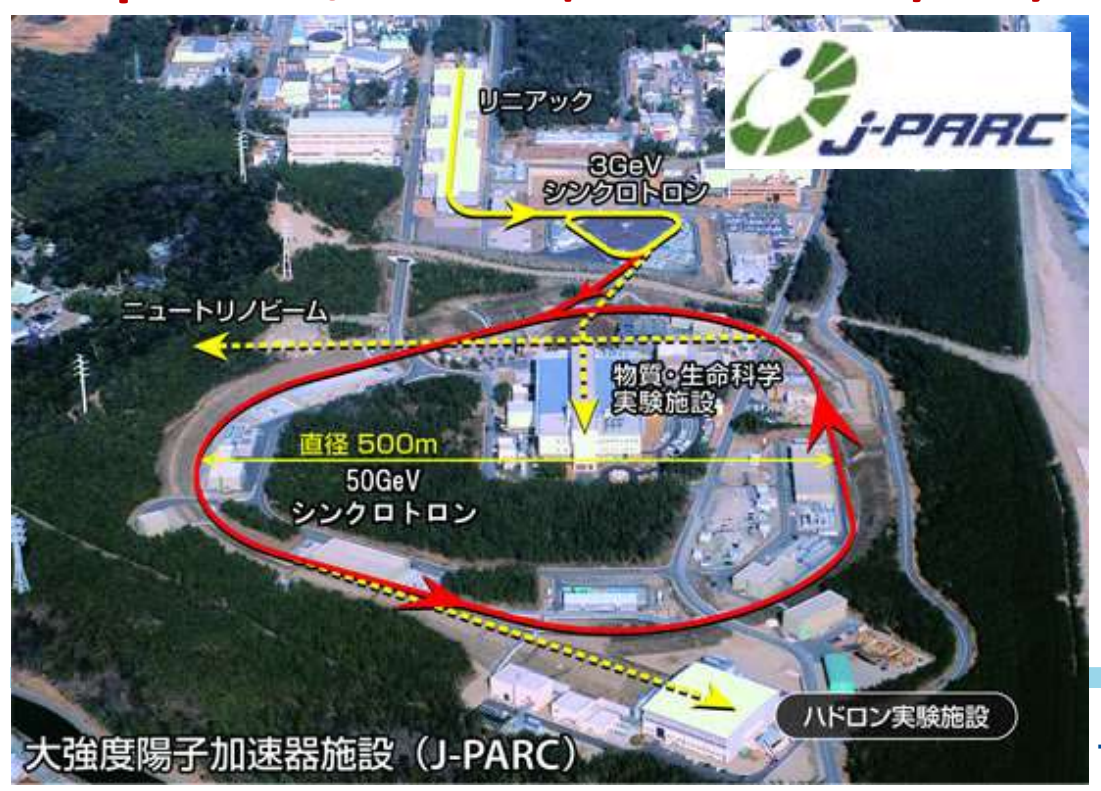

Fermilab Proton Accelerator Complex 8/120 GeV (810 km to MINOS)

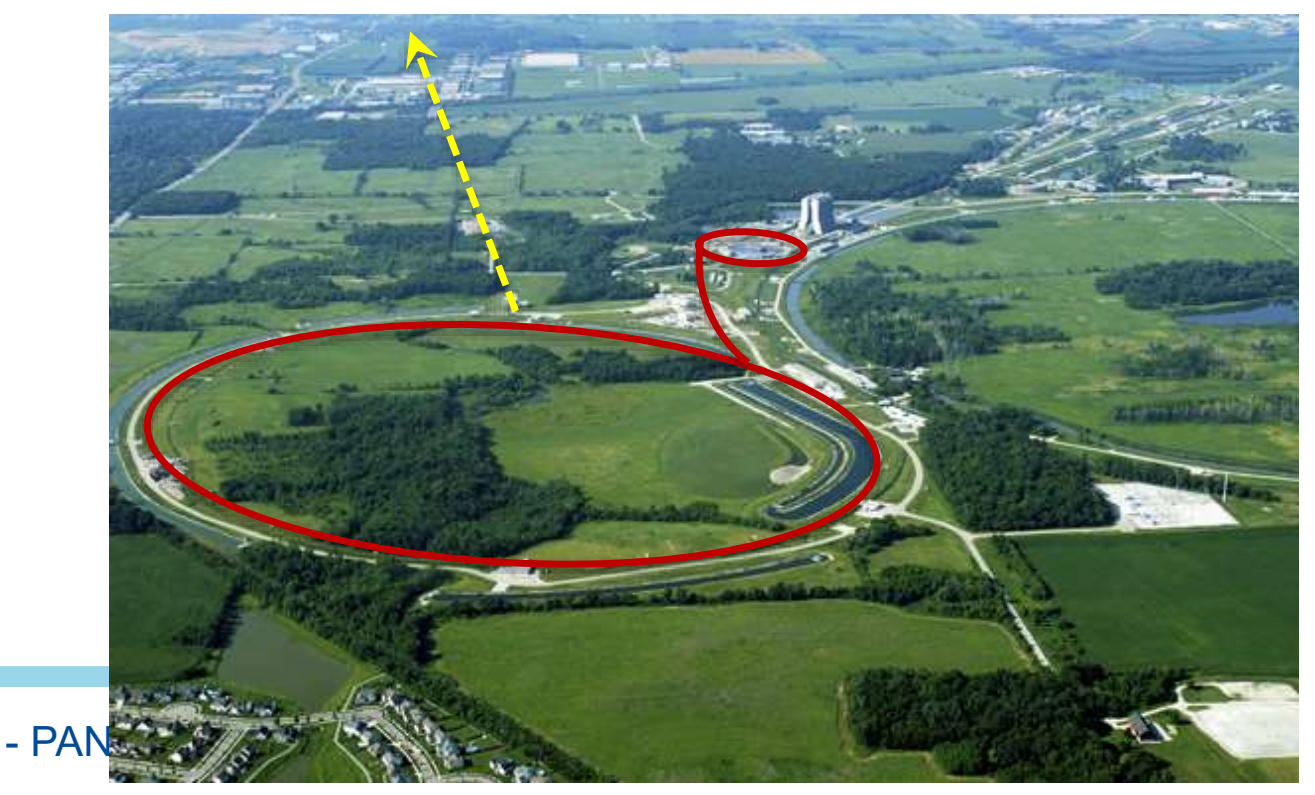




\section{Fermilab and J-PARC Power Upgrades}

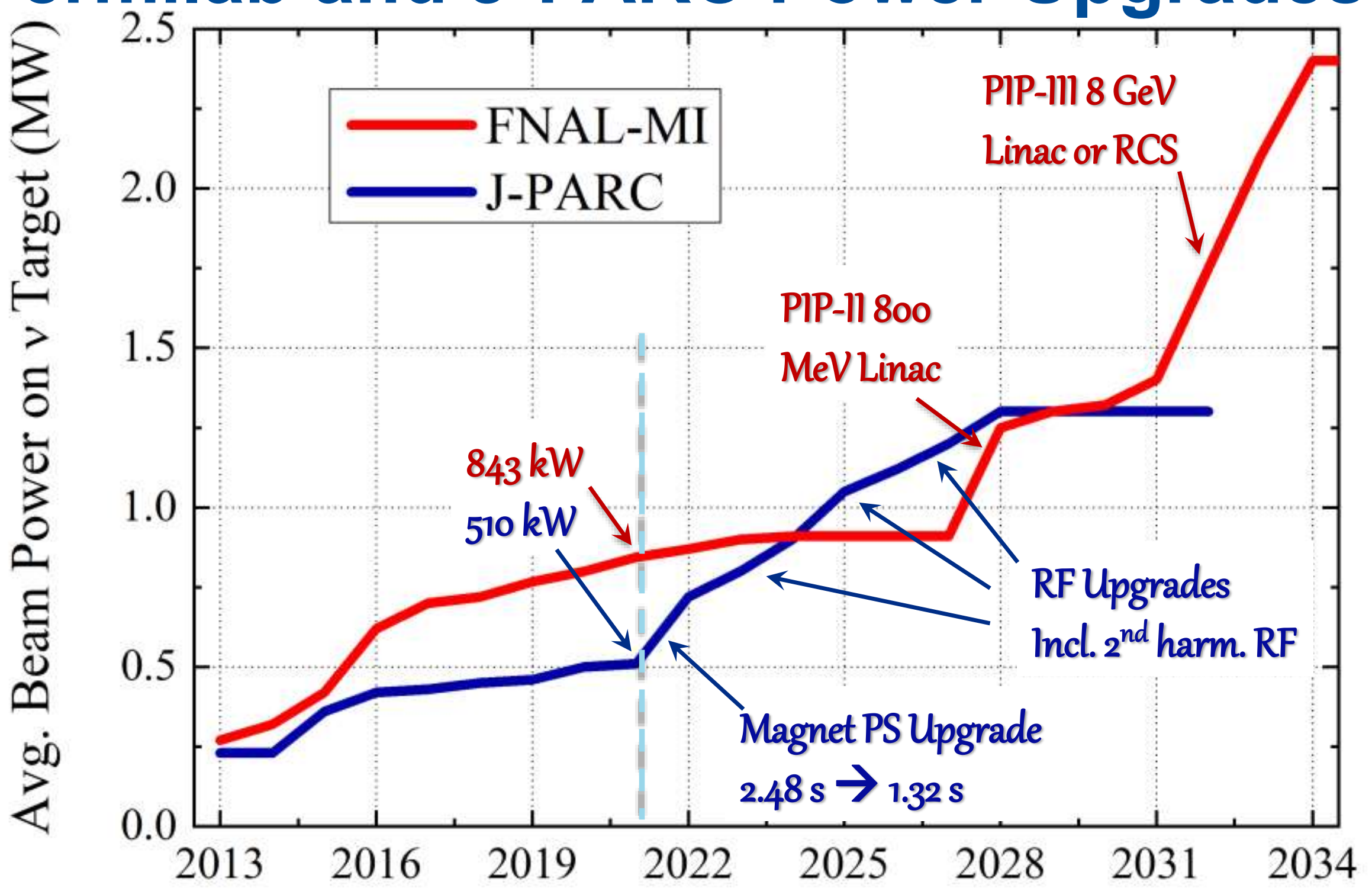




\section{Muon g-2 - 2021 !}

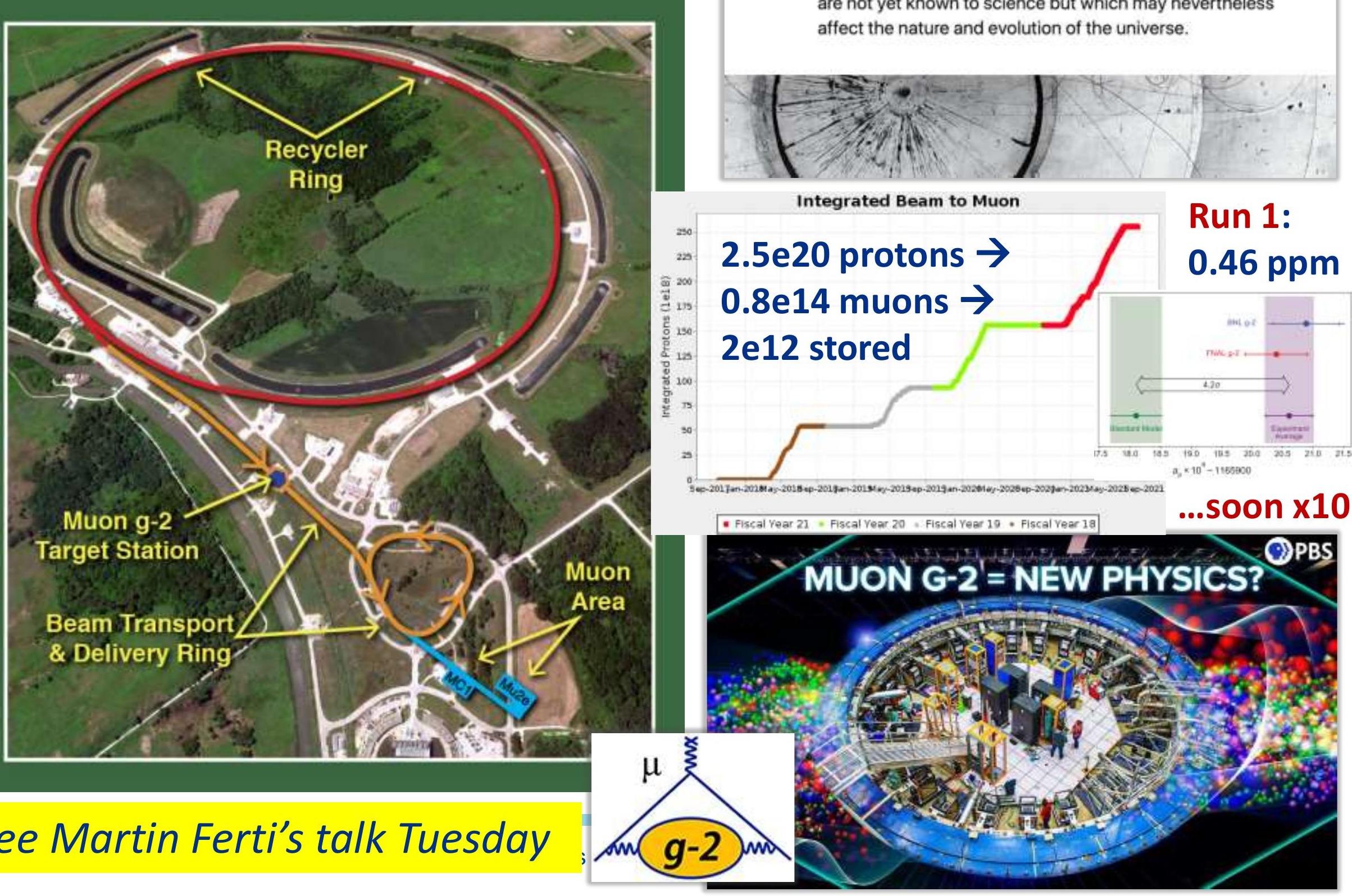


HEP Colliders

Seven in operation now

\begin{tabular}{lccccc}
\hline \hline & Species & $E_{b}(\mathrm{GeV})$ & $C(\mathrm{~m})$ & $\mathcal{L}_{\text {peak }}^{\max }$ & Years \\
\hline VEPP-4M & $e^{+} e^{-}$ & 6 & 366 & $2 \times 10^{31}$ & 1979 -present \\
BEPC-I/II & $e^{+} e^{-}$ & 2.3 & 238 & $10^{33}$ & 1989 -present \\
DAФNE & $e^{+} e^{-}$ & 0.51 & 98 & $4.5 \times 10^{32}$ & 1997 -present \\
RHIC & $p, i$ & 255 & 3834 & $2.5 \times 10^{32}$ & 2000 -present \\
LHC & $p, i$ & 6500 & 26659 & $2.1 \times 10^{34}$ & 2009 -present \\
VEPP2000 & $e^{+} e^{-}$ & 1.0 & 24 & $4 \times 10^{31}$ & 2010 -present \\
S-KEKB & $e^{+} e^{-}$ & $7+4$ & 3016 & $8 \times 10^{35}$ & 2018-present \\
& & & v. Sthilser and F. Zimmermant: Modem and future collidicr
\end{tabular}

Highlights - LHC : pp 13 $\rightarrow 14$ TeV cme

- $190 \mathrm{fb}-1 / \mathrm{IP}$ by now, $\mathrm{x} 2$ design luminosity

- High-Lumi upgrade by 2028: double beam current, smaller $\beta^{*}$ (new $\mathrm{Nb}_{3} \mathrm{Sn}$ IR magnets), "crabbing", leveling @14 TeV $\rightarrow 250 \mathrm{fb}^{-1} / \mathrm{yr}$

- Followed by $\sim$ decade of ops to 3-4 $\mathrm{ab}^{-1}$

Highlights - Super-KEKB: e+e- 7+4 GeV

- Startup in 2018, world record $L=3.1 \mathrm{e} 34 \mathrm{~cm}^{-2} \mathrm{~s}^{-1}$

- Design luminosity goal x40 of KEK-B

- Now $\sim 4 \%$ of the goal, steady progress

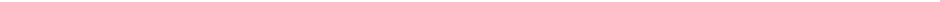


Future Collider Proposals: 8 Higgs/EW factories

\begin{tabular}{l|l|}
\hline Name & Details \\
\hline CepC & $e+e-, \sqrt{s}=0.24 \mathrm{TeV}, \mathrm{L}=3.0 \times 10^{34}$ \\
\hline CLIC (Higgs factory) & $e+e-, \sqrt{s}=0.38 \mathrm{TeV}, \mathrm{L}=1.5 \times 10^{34}$ \\
\hline ERL ee collider & $e+e-, \sqrt{s}=0.24 \mathrm{TeV}, \mathrm{L}=73 \times 10^{34}$ \\
\hline FCC-ee & $e+e-, \sqrt{s}=0.24 \mathrm{TeV}, \mathrm{L}=17 \times 10^{34}$ \\
\hline gamma gamma & $\mathrm{x}-\mathrm{ray} \mathrm{FEL}-\mathrm{based} \gamma \gamma$ collider \\
\hline ILC (Higgs factory) & $e+e-, \sqrt{s}=0.25 \mathrm{TeV}, \mathrm{L}=1.4 \times 10^{34}$ \\
\hline LHeC & $e p, \sqrt{s}=1.3 \mathrm{TeV}, \mathrm{L}=0.1 \times 10^{34}$ \\
\hline MC (Higgs factory) & $\mu \mu, \sqrt{s}=0.13 \mathrm{TeV}, \mathrm{L}=0.01 \times 10^{34}$ \\
\hline
\end{tabular}

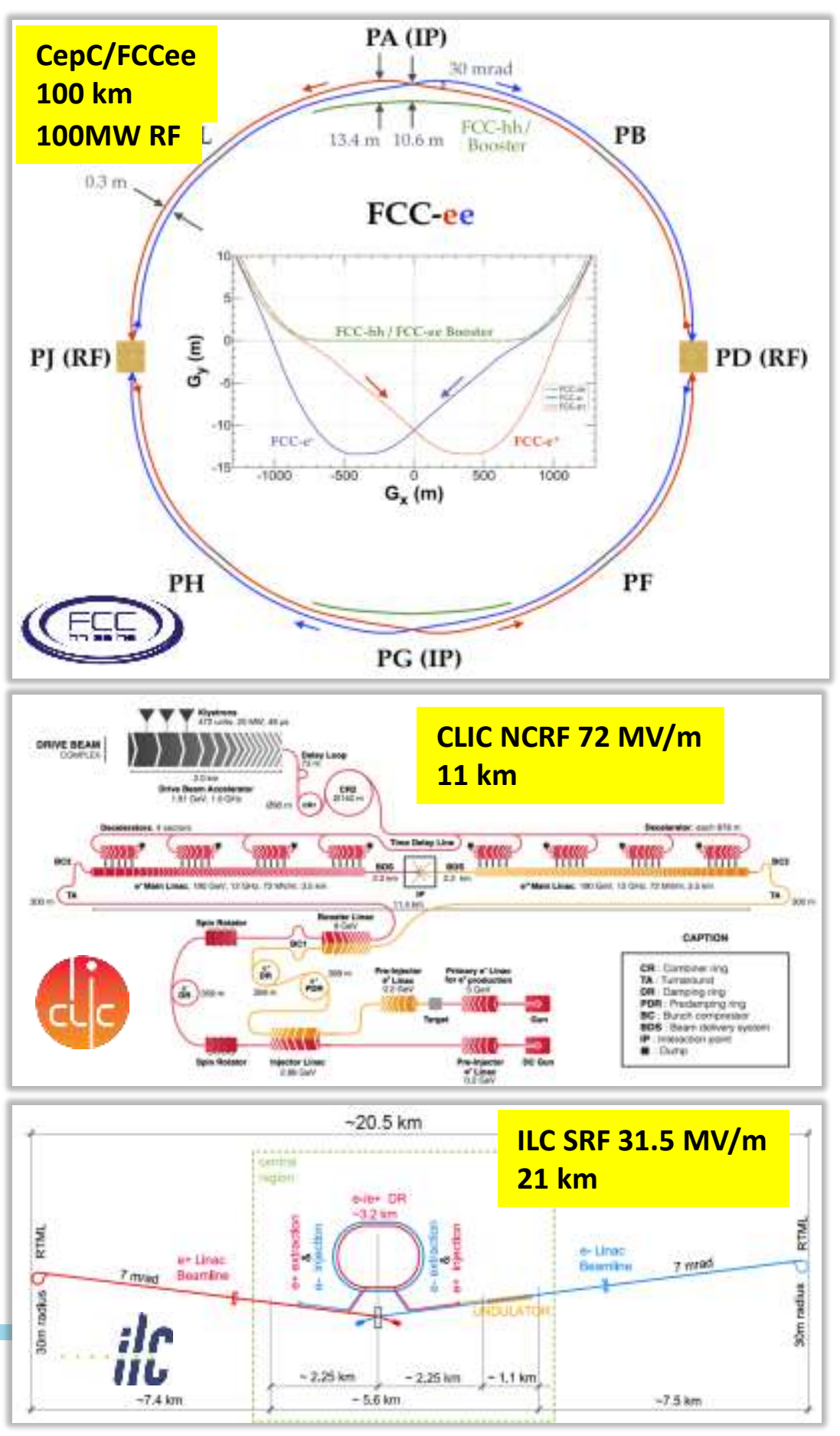




\section{7 (!) High Energy Collider Concepts/Proposals}

\section{Name}

Cryo-Cooled Copper linac

High Energy CLIC

High Energy ILC

FCC-hh

SPPC

Collider-in-Sea

LHeC

FCC-eh

CEPC-SPPpC-eh

VHE-ep

MC - Proton Driver 1

MC - Proton Driver 2

MC - Proton Driver 3

MC - Positron Driver

LWFA-LC (e+e- and $\gamma \gamma$ )

PWFA-LC (e+e- and $\gamma \gamma)$

SWFA-LC

\section{Details}

$e+e-, \sqrt{s}=2 \mathrm{TeV}, \mathrm{L}=4.5 \times 10^{34}$

$e+e-, \sqrt{s}=1.5-3 \mathrm{TeV}, \mathrm{L}=5.9 \times 10^{34}$

$\mathrm{e}+\mathrm{e}-, \sqrt{s}=1-3 \mathrm{TeV}$

pp, $\sqrt{s}=100 \mathrm{TeV}, \mathrm{L}=30 \times 10^{34}$

pp, $\sqrt{s}=75 / 150 \mathrm{TeV}, \mathrm{L}=10 \times 10^{34}$

$\mathrm{pp}, \sqrt{\mathrm{s}}=500 \mathrm{TeV}, \mathrm{L}=50 \times 10^{34}$

ep, $\sqrt{s}=1.3 \mathrm{TeV}, \mathrm{L}=1 \times 10^{34}$

ep, $\sqrt{s}=3.5 \mathrm{TeV}, \mathrm{L}=1 \times 10^{34}$

$e p, \sqrt{s}=6 \mathrm{TeV}, \mathrm{L}=4.5 \times 10^{33}$

$e p, \sqrt{s}=9 \mathrm{TeV}$

$\mu \mu, \sqrt{s}=1.5 \mathrm{TeV}, \mathrm{L}=1 \times 10^{34}$

$\mu \mu, \sqrt{s}=3 \mathrm{TeV}, \mathrm{L}=2 \times 10^{34}$

$\mu \mu, \sqrt{s}=10-14 \mathrm{TeV}, \mathrm{L}=20 \times 10^{34}$

$\mu \mu, \sqrt{s}=10-14 \mathrm{TeV}, \mathrm{L}=20 \times 10^{34}$

Laser driven; e+e-, $\sqrt{s}=1-30 \mathrm{TeV}$

Beam driven; e+e-, $\sqrt{s}=1-30 \mathrm{TeV}$

Structure wakefields; e+e-, $\sqrt{s}=1-30$ $\mathrm{TeV}$
CLIC e+e- 3 TeV, $100 \mathrm{MV} / \mathrm{m} 50 \mathrm{~km}$

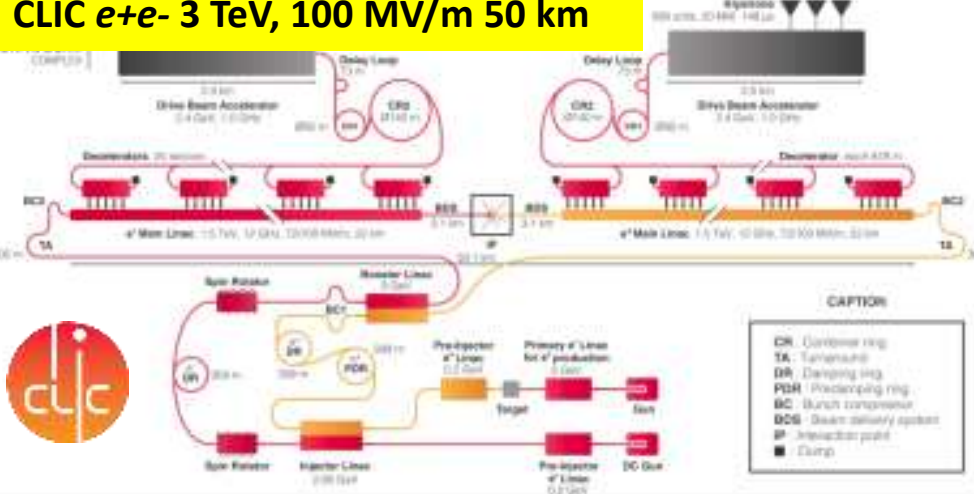

pp 100 km : SPPC 75 TeV, 12 T magnets, FCChh 100/16 T

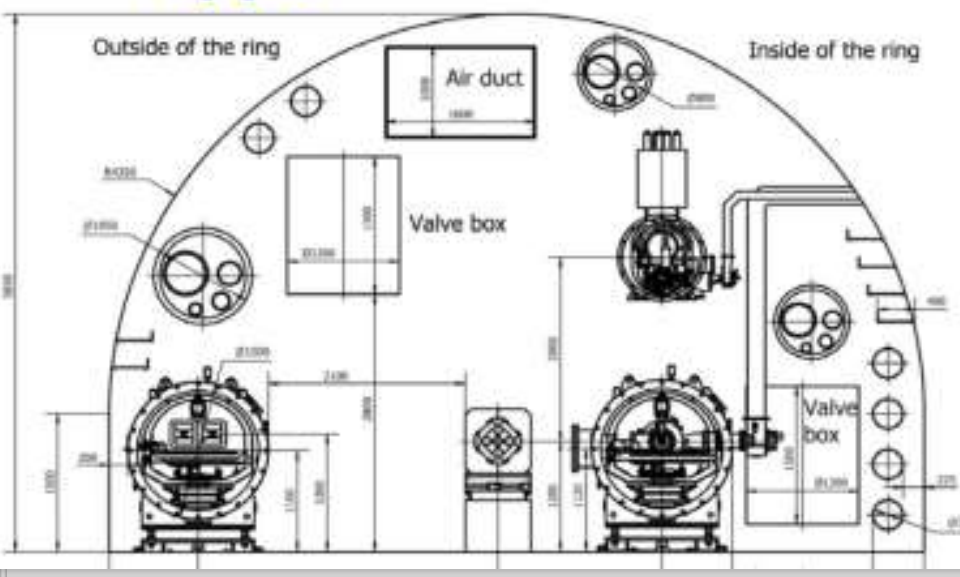

$\mu+\mu$ - 10-14 TeV cme 10-14 km, $16 \mathrm{~T}$ magnets

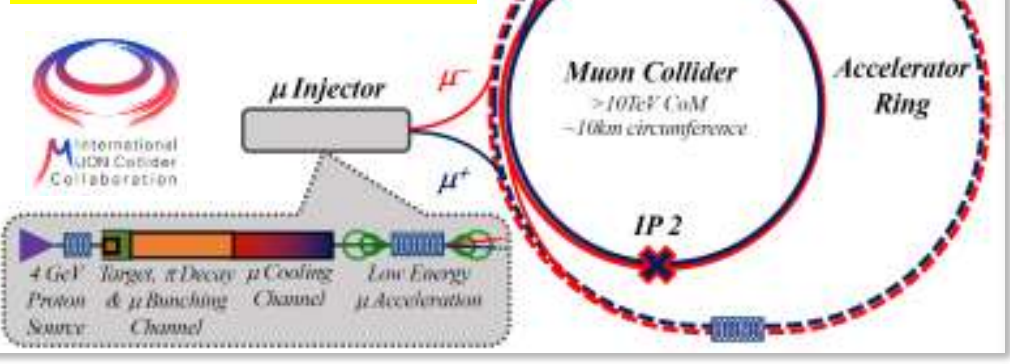



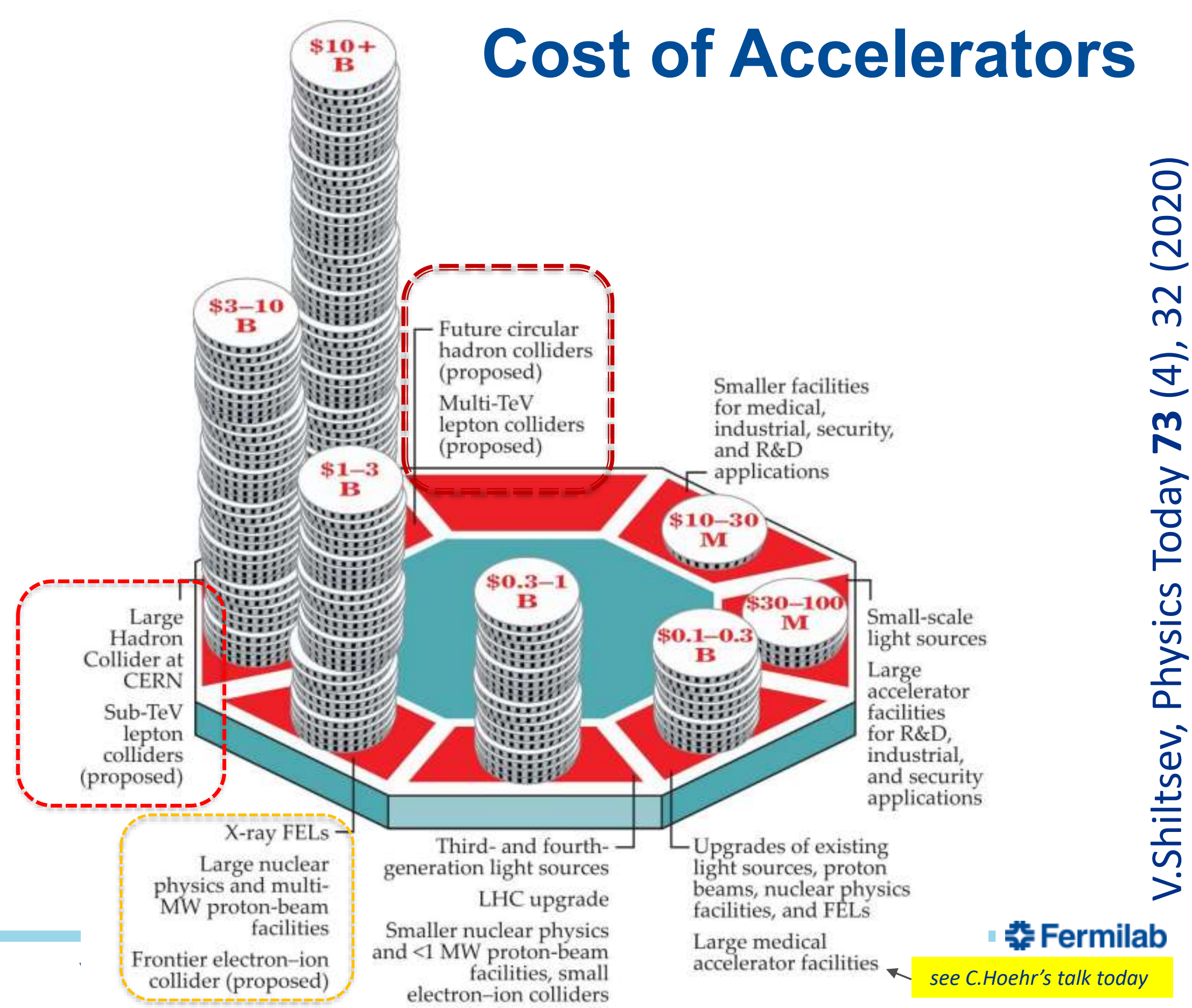
Cost is set by the scale (energy, length, power) and technology

Normal Conducting Magnets

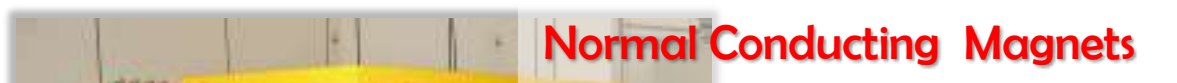

- Accelerator technology $\sim 50 \pm 10 \%$ (magnets NC and SC, RF and SCRF)

- Civil construction technology $\sim 35 \pm 15 \%$

- Power production, delivery and distribution technology

$\sim 15 \pm 10 \%$ 
State of the art NC (warm) magnets for $4^{\text {th }}$ gen light sources :

- high quality

- buy from industry

\section{NC RF:}

- $28 \mathrm{MV} / \mathrm{m}$ in

SwissFEL ('17)

- 100MV/m in CLIC structures

- Aim for $117 \mathrm{MV} / \mathrm{m}$ in cold copper $\left(\mathrm{LN}_{2}\right)$ structures (SLAC)
Supercond. magnets, for colliders and undulators:

- 8.3T in LHC - 14.5T by US MDP (2020)

- $290 \mathrm{~T} / \mathrm{s}$ fast cycling HTS (FNAL, 2021)

SC RF:

- $25 \mathrm{MV} / \mathrm{m}$ at $Q_{0}=1 \mathrm{e} 10$ at $1.3 \mathrm{GHz}$ EurXFEL - ILC specs $31.5 \mathrm{MV} / \mathrm{m}$ at FNAL - Nitrogen doping $\rightarrow Q_{0} \sim 3 \mathrm{e} 10$ - Aim at $~ 50 \mathrm{MV} / \mathrm{m}$ in $1.3 \mathrm{GHz}$ 


\section{Beam Cooling}

Beam Phase Space Density Increase

- As needed for a collider

$$
\mathcal{L}=f_{\operatorname{coll}} \frac{N_{1} N_{2}}{4 \pi \sigma_{x}^{*} \sigma_{y}^{*}}
$$

- Forbidden by the Liouville theorem in non-dissipative systems
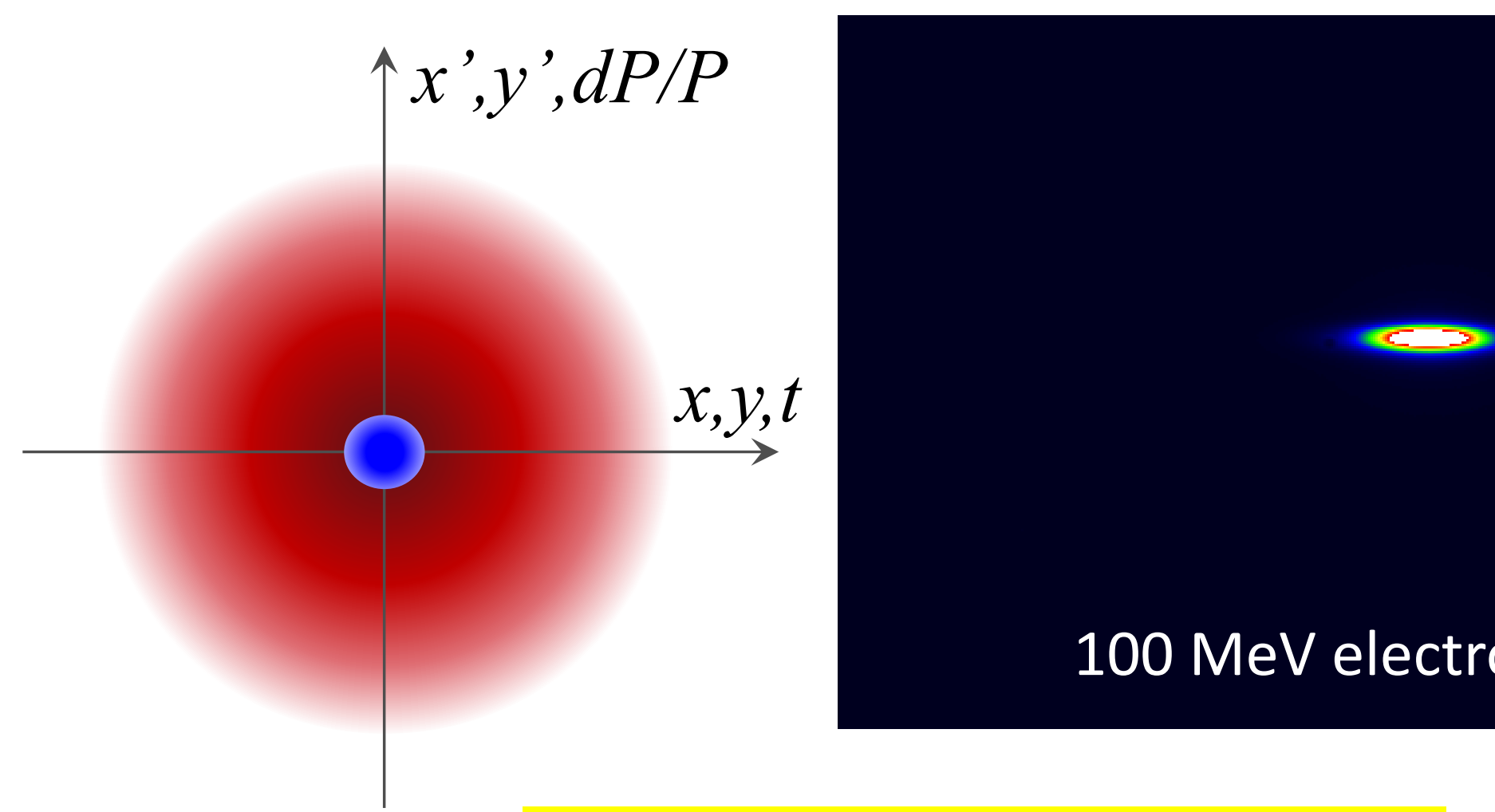

$100 \mathrm{MeV}$ electrons in IOTA ring 


\section{Beam Cooling Methods to Date}

Synchrotron Radiation Damping - since 1960's

- common in all e+/e- rings

Electron Cooling - since 1970's

- Widely used to cool ions and antiprotons

- 0.1 - $8 \mathrm{GeV} / \mathrm{n}$ (50 keV - $4 \mathrm{MeV}$ electrons DC)

Stochastic Cooling - since 1970's

- Widely used to cool ions and antiprotons

- $0.1-100 \mathrm{GeV} / \mathrm{n}$ (up to $10 \mathrm{GHz}$ feedback BW)

Laser Cooling - since 1990's $\Omega=\gamma \omega_{21}(1-\beta \cos \theta)$

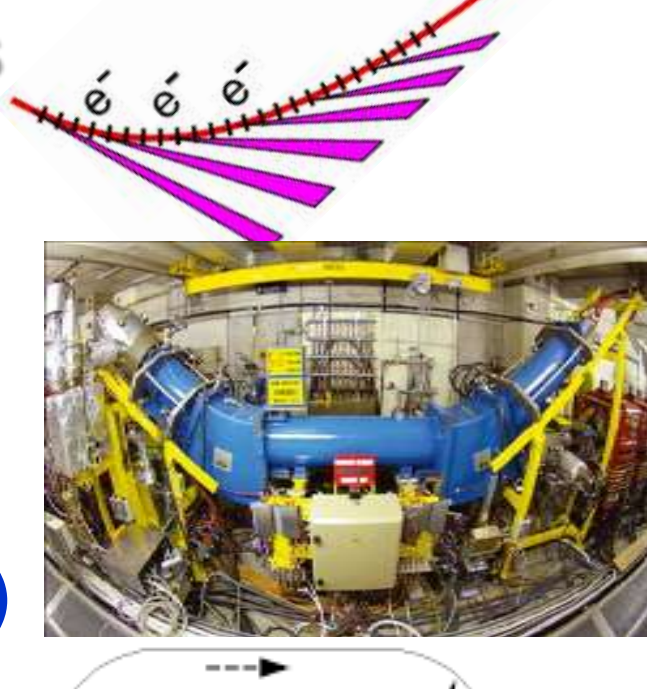

- Works for some highly charged ions

- 0.1-0.5 GeV/n, deep cooling, spectroscopy 


\section{Beam Cooling Breakthroughs}

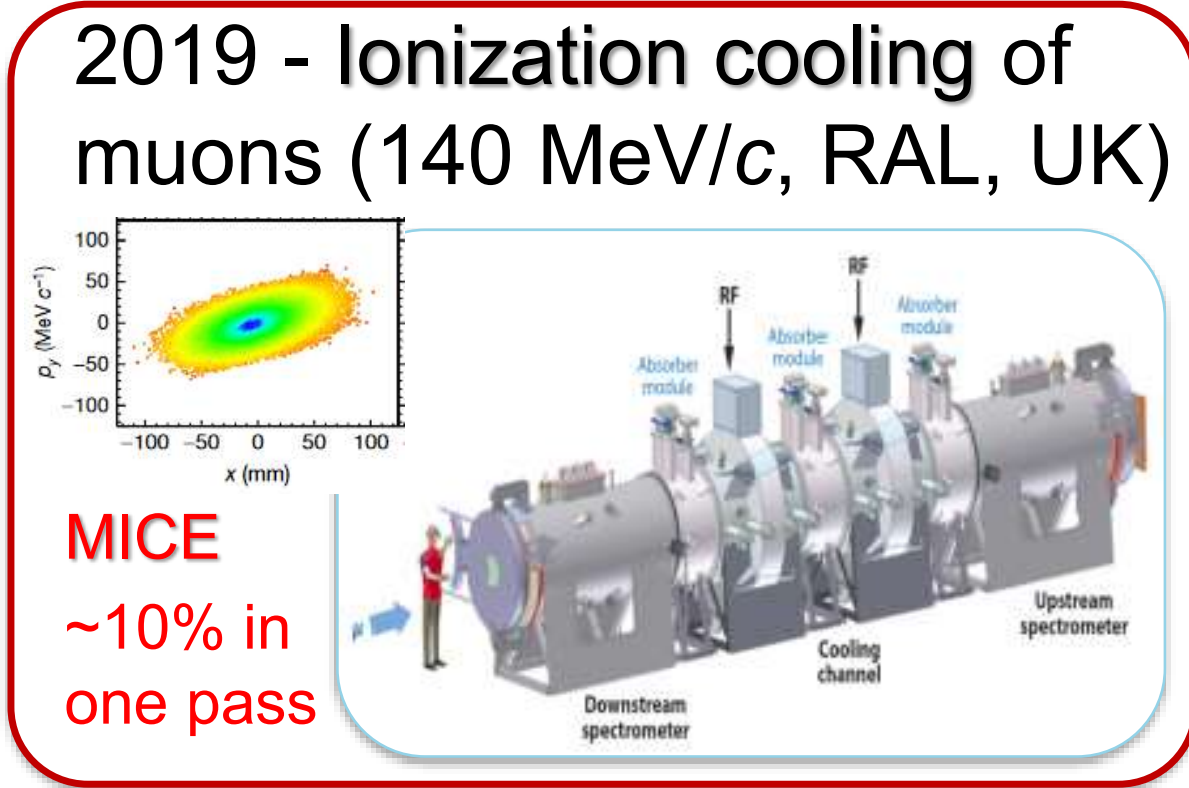

2020 - "Bunched" electron cooling of ions $(\gamma \sim 5, \mathrm{BNL})$

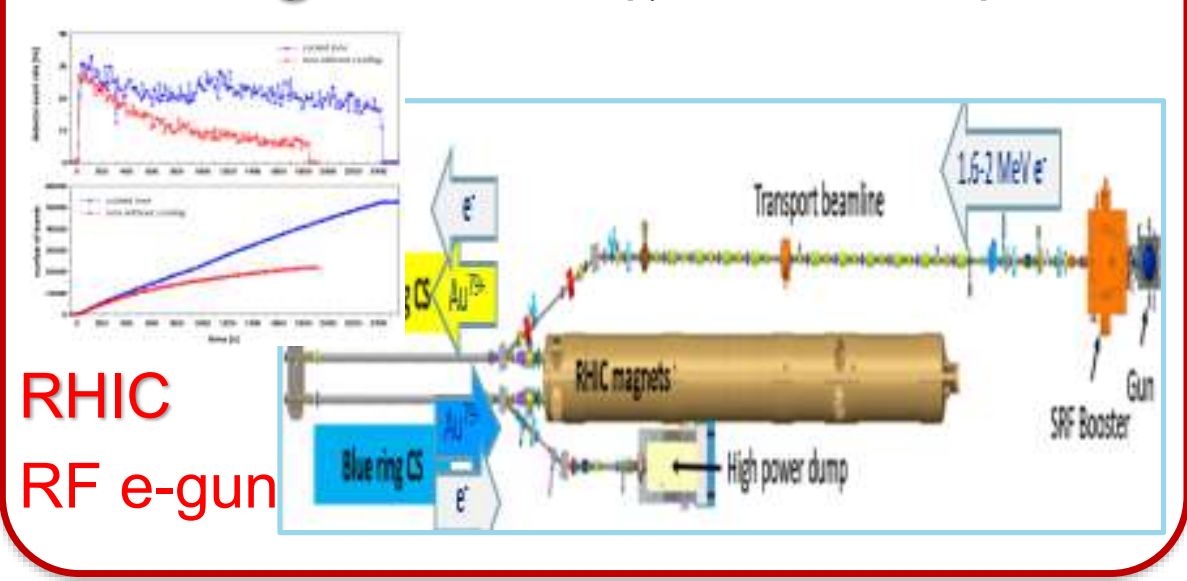

2021 - Coherent Electron cooling of ions $(26.5 \mathrm{GeV} / n$, RHIC) - ongoing PoP exp't at BNL

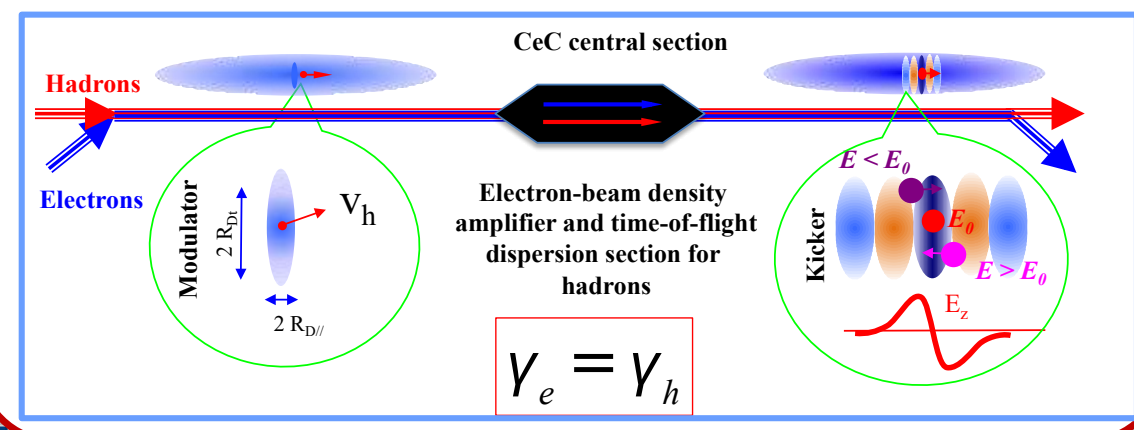

2021 - Optical Stochastic cooling e- (100 MeV, FNAL)

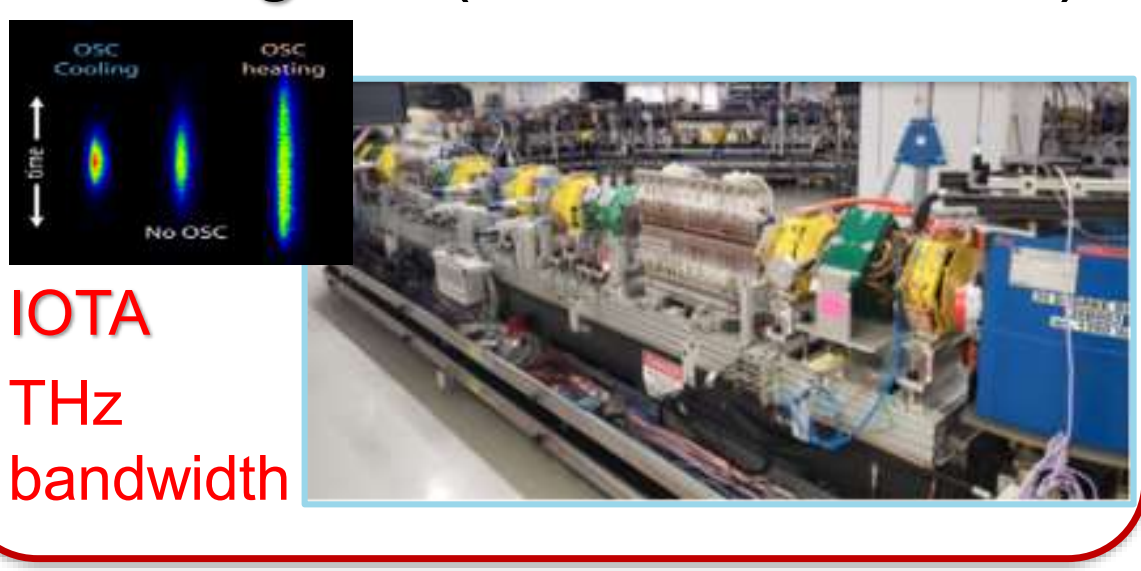




\section{Acceleration in Plasma}

Plasma waves can sustain high fields:

$E_{0}=\frac{m_{e} c \omega_{p}}{e} \approx 100\left[\frac{G e V}{m}\right] \cdot \sqrt{n_{0}\left[10^{18} \mathrm{~cm}^{-3}\right]}$

Excitation by (smth short and powerful):

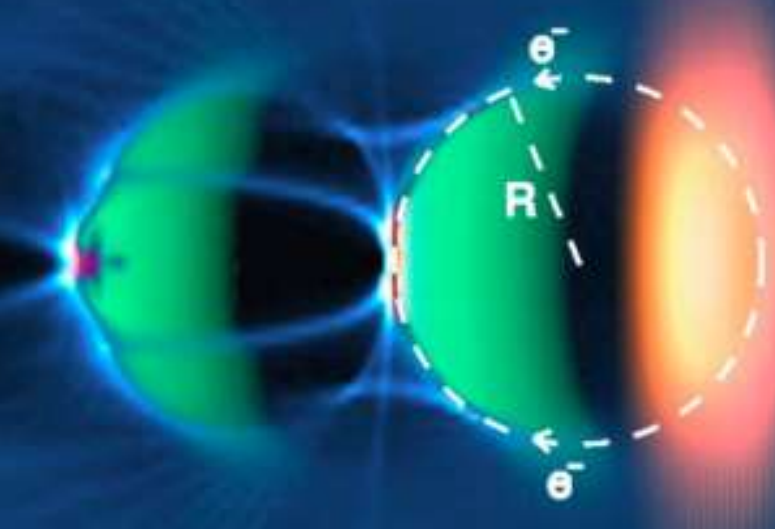
laser $4.3 \mathrm{GeV}\left(10^{18} \mathrm{~cm}^{-3} 9 \mathrm{~cm}\right) e$ - bunch $9 \mathrm{GeV}\left(\sim 10^{17} \mathrm{~cm}^{-3} 1.3 \mathrm{~m}\right) p+$ bunch $2 \mathrm{GeV}\left(\sim 10^{15} \mathrm{~cm}^{-3} 10 \mathrm{~m}\right)$
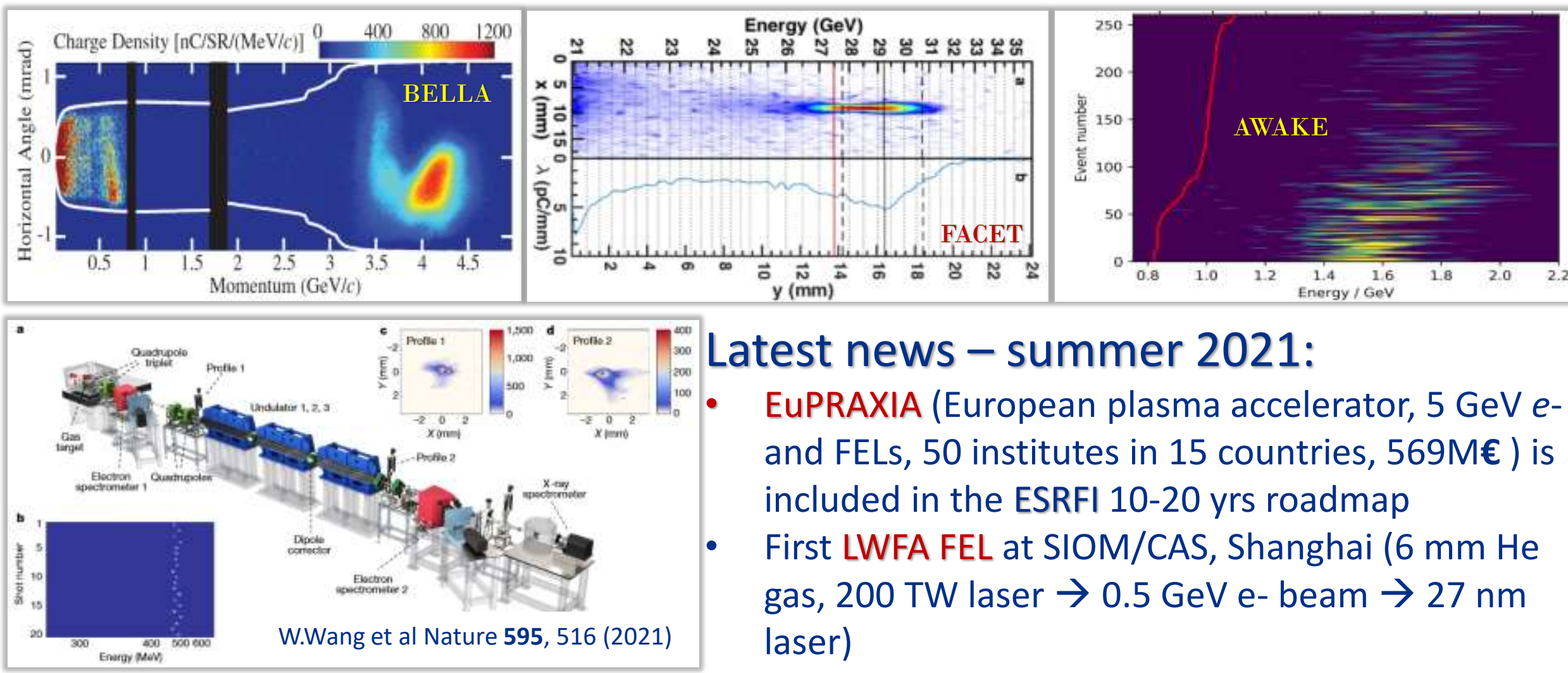

Latest news - summer 2021:

- EuPRAXIA (European plasma accelerator, $5 \mathrm{GeV} e$ and FELs, 50 institutes in 15 countries, 569M $€$ ) is included in the ESRFI $10-20$ yrs roadmap

- First LWFA FEL at SIOM/CAS, Shanghai $(6 \mathrm{~mm}$ He gas, 200 TW laser $\rightarrow 0.5 \mathrm{GeV}$ e- beam $\rightarrow 27 \mathrm{~nm}$ laser) 


\section{Take Away Message on Accelerators:}

- Remarkable progress over the past 4 years:

- Accelerators for NP, BES, neutrinos and rare processes, colliders

- e.g., FRIB, XFELs, China SNS, power records at FNAL and JPARC, luminosity records at LHC and SuperKEKB

- Physics of beams breakthroughs

- Several new beam cooling schemes, plasma acceleration to $\mathrm{O}(5 \mathrm{GeV})$ - with beams good enough for FELs

- Core technology advances

- Records in RF gradients, $B$-field, $d B / d t$ rate, MWs beam targets

- Bright future ahead:

- Next: NICA, XFELs, High Lumi LHC, PIP-II, ESS, FAIR, etc

- Future: Higgs factories (linear or circular), Multi-TeV colliders $(p p, \mu \mu, e e)$ 


\section{Thanks for your attention!}

\section{Some references}

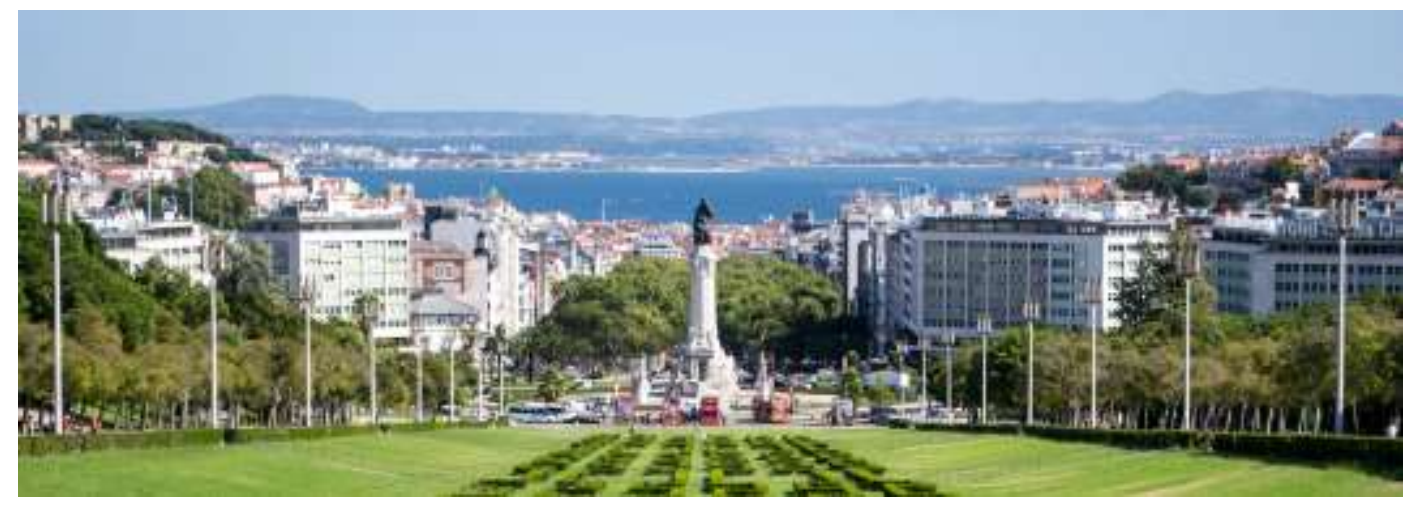

- Accelerators - V.Shiltsev, Physics Today 73(44), 32 (2020)

- XFELs - I.Georgescu Nature Reviews Physics 2, 345 (2020)

- Modern and Future Colliders - V. Shiltsev, F. Zimmermann Rev. Mod. Phys. 93, 015006 (2021)

- RHIC lumi - https://www.rhichome.bnl.gov/RHIC/Runs/

- LHC ions - J.Jowett, IPAC'2019

- MICE - M.Bogomilov et al Nature, 578(7793): 53-59 (2020)

- Can the US compete in BES? - https://science.osti.gov//media/bes/besac/pdf/Reports/AH DOE2021-Benchmarking 202108.pdf 


\section{Acknowledgements}

Input/useful discussions with:

Frank Zimmermann (CERN)

Steve Gourlay(LBNL)

Tor Raubenheimer (SLAC)

Dmitri Denisov (BNL)

Meenakshi Narain (UB)
"Modern and Future Colliders" (Rev.Mod.Phys.)

\section{EPPSU-Snowmass'21 input from:}

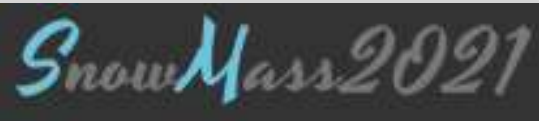

M.Benedikt (CERN), P.Bhat (FNAL), C.Biscari (ALBA), A.Blondel (CERN), J.Brau (UO), O.Bruning(CERN), A.Canepa (FNAL), W.Chou (IHEP, China), J.P.Delahaye (CERN), D.Denisov (BNL), V.Dolgashev (SLAC), E.Gschwendtner (CERN), A.Grasselino (FNAL), M.Klein (CERN), W.Krasny (CNRS), M.Lamont (CERN), W.Leemans (DESY), E.Levichev (BINP), V.Litvinenko (SUNY), K.Long (ICL), D.Lucchesi (INFN), B.List (DESY), H.Montgomery (JLab), P.Muggli (MPG), D.Neuffer (FNAL), H.Padamsee (Cornell), M.Palmer (BNL), N.Pastrone (INFN), H.Piekarz (FNAL), Q.Qin (IHEP), L.Rivkin (EPFL/PSI), A.Romanenko (FNAL), T.Roser (BNL), M.Ross (SLAC), D.Schulte (CERN), S.Seletsky (BNL), A.Seryi (Jlab), T.Sen (FNAL), A.Valishev (FNAL), F.Willeke (BNL), A.Yamomoto (KEK), V.Yakovlev, A.Zlobin (FNAL) 